\title{
1 A global multiregional proteomic map of the human cerebral cortex
}

2 Zhengguang Guo ${ }^{1 \dagger}$, Chen Shao ${ }^{2 \dagger}$, Yang Zhang ${ }^{3 \dagger}$, Wenying $\mathrm{Qiu}^{4 \dagger}$, Wenting $\mathrm{Li}^{4}$, Weimin

$3 \mathrm{Zhu}^{2}$, Qian Yang ${ }^{4}$, Yin Huang ${ }^{2}$, Lili Pan ${ }^{2}$, Yuepan Dong ${ }^{2}$, Haidan Sun ${ }^{1}$, Xiaoping Xiao ${ }^{1}$,

Wei Sun ${ }^{1 *}$, Chao $\mathrm{Ma}^{4 *}$, Liwei Zhang ${ }^{3 *}$

$5{ }^{1}$ Core Facility of Instrument, Institute of Basic Medical Sciences, Chinese Academy of

6 Medical Sciences/School of Basic Medicine, Peking Union Medical College, Beijing,

7

8

9

100005, China.

${ }^{2}$ State Key Laboratory of Proteomics, Beijing Proteome Research Center, National

Center for Protein Sciences (Beijing), Beijing Institute of Lifeomics, Beijing 102206,

\section{China}

${ }^{3}$ Department of Neurosurgery, China National Clinical Research Center for Neurological Diseases, Beijing Tiantan Hospital, Capital Medical University, Beijing, 100050, China.

${ }^{4}$ Department of Human Anatomy, Histology, and Embryology, Institute of Basic

Medical Sciences, Neuroscience Center, Chinese Academy of Medical Sciences, School of Basic Medicine, Peking Union Medical College, Beijing, China.

${ }^{\text {\#Wei Sun: sunwei1018@hotmail.com }}$

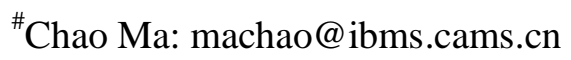

"Liwei Zhang: zhangliweittyy@163.com

${ }^{\dagger}$ Contributed equally

\section{Running title: Proteomic map of the human cerebral cortex}




\section{Abstract}

The Brodmann area (BA)-based map is one of the most widely used cortical maps for studies of human brain functions and in clinical practice; however, the molecular architecture of BAs remain unknown. The present study provided a global multiregional proteomic map of the human cerebral cortex by analyzing 29 BAs. These 29 BAs were grouped into 6 clusters based on similarities in proteomic patterns: the motor and sensory cluster, vision cluster, auditory cluster and Broca's area, Wernicke's area cluster, cingulate cortex cluster, and heterogeneous function cluster. We identified 474 cluster-specific and 134 BA-specific signature proteins whose functions are closely associated with specialized functions and disease vulnerability of the corresponding cluster or BA. The findings of the present study could provide explanations for the functional connections between the anterior cingulate cortex and sensorimotor cortex and for anxiety-related function in the sensorimotor cortex. The brain transcriptomic and proteomic comparison indicated that they both could reflect the function of cerebral cortex,but showed different characteristics. These proteomic data are publicly available at the Human Brain Proteome Atlas (www.brain-omics.com). Our results may enhance our understanding of the molecular basis of brain functions and provide an important resource to support human brain research.

\section{Key words}

Brodmann areas; proteomics; cerebral cortex; cingulate cortex; sensorimotor cortex 


\section{Introduction}

The human cerebral cortex, the outer layer of the cerebrum, exhibits great complexity in its histological structure and cellular organization ${ }^{1}$. In 1909, Korbininan Brodmann distinguished a total of 52 subareas based on the differences in cytoarchitectural organization across the cortex; these areas were later termed Brodmann areas (BAs) ${ }^{2}$. Subsequently, BA-based map has become one of the most widely used cortical maps for the investigation of the brain functions and in clinical practice. BAs are often correlated with certain cortical functions. For example, Broca's speech and language area is consistently localized in BAs 44 and 45 . cytoarchitecture ${ }^{1,2}$, imaging ${ }^{1,3}$, and functional roles ${ }^{3}$ of BAs have been extensively studied, and comprehensive human brain MRI imaging ${ }^{1}$, histology ${ }^{1}$, and connectome maps ${ }^{3}$ have been investigated.

Molecular annotations, especially at the protein level, of various BAs will provide a deeper understanding of the molecular basis for diverse brain functions. Transcriptomic and proteomic maps of the brain have been generated to study the molecular basis of distinct cytoarchitecture and functions of various BAs ${ }^{1}$. The Allen Institute for Brain Science has generated a transcriptional atlas of the human brain by comprehensive profiling of nearly 900 anatomically precise subdivisions using microarrays ${ }^{1}$. The human neocortex is characterized by a relatively homogeneous transcriptional pattern; the molecular topography of the neocortex is similar to its cytoarchitecture. At the protein level, several studies generated a protein atlas of the mouse brain and identified some brain region-restricted proteins ${ }^{4,5}$ or cell type-specific proteins ${ }^{4}$. In 2014, Kim et al drafted a proteomic profile of the human frontal cortex from a pool of three individuals and identified 9,060 proteins ${ }^{6}$. In 2017, Becky el al. ${ }^{7}$ performed a proteomic survey of 7 postnatal human brain regions, including two cerebral cortex regions, the cerebellar cortex, the hippocampus, and other neurological nuclei, ranging from early infancy to adulthood. Individual variations due to age and sex were considerably lower than variations between brain 
regions. Importantly, the differences in brain cytoarchitecture, development, and function are better represented by changes at the protein level than by changes at the transcriptional level.

Previous omics studies have increased our understanding of the brain function. However, some important scientific aspects have not been investigated. Functional annotation of the brain demonstrated that several brain regions are functionally connected but are not spatially adjacent, such as BA41/42 (auditory cortex) and BA $44 / 45$ (Broca's area and language production) ${ }^{3,8}$. However, the data on the brain transcriptome showed a relatively homogeneous expression pattern in the neocortex, and molecular topography in this area is similar to spatial topography. Proteins are the main functional operators in all cells. Proteomic map of the cerebral cortex may provide new insight into this scientific context. Previous studies of the brain proteome investigated only a few BAs, which are not sufficient to illustrate these aspects. Thus, a comprehensive proteomic map of various BAs of the human cerebral cortex is urgently needed.

The present study used 29 BA postmortem specimens to generate a global multiregional proteomic map of the human cerebral cortex. Initially, an extensive proteomic library of BA46 was generated. Then, 29 BAs were quantitatively analyzed using the 2DLC/MS/MS approach combined with isobaric tags for relative and absolute quantitation (iTRAQ) labeling. Clustering analysis of 29 BAs was performed based on the similarities in protein expression patterns. Cluster- and BA- signature proteins were identified and analyzed using bioinformatics. The major functional brain areas, including the cingulate cortex, motor cortex, sensory cortex, language area, and visual cortex, were functionally annotated using proteomic data. The transcriptomes of above BAs were also analyzed and the comparison of proteome and transcriptome on BA functions was presented. The results of the present study provide a molecular basis for BA functions. Furthermore, the database provides an important resource to support brain research. 
104

105

106

107

108

109

110

111

112

113

114

115

116

117

118

119

120

121

122

123

124

125

126

127

128

129

130

\section{Results}

\section{A broad and comprehensive atlas of brain proteome}

A single human brain specimen was obtained from the Human Brain Bank facilitated by the China Human Brain Bank Consortium. The brain specimen was collected from a 54-year-old willed male donor who died of lung cancer and did not have any history of brain metastasis, trauma, or other diseases. The samples of 29 BAs of the left cerebral hemisphere were collected with a postmortem delay of less than 6 hours. Hematoxylin-eosin staining (HE staining) of the samples showed that the brain was cancer-free (Supplementary Figure 1). The protocols for brain tissue acquisition, dissection, and sample preparation were under consistent and stringent quality control (see Materials and Methods) based on the standardized operational protocol (SOP) of the Human Brain Bank of China ${ }^{9}$.

BA46 (dorsolateral prefrontal cortex (DFC)) played an important role in many cognitive processes and had been comprehensively characterized in previous proteomic studies ${ }^{6,7}$. Therefore, deep proteomic profiling of BA 46 was performed using the 2DLC/MS/MS approach (Figure 1A) to construct a Chinese reference brain protein library. A total of 8,600 proteins (Supplementary Table 1 A-B, data quality in Supplementary Figure 2A) were identified in the present study, corresponding to $73 \%$ and $89 \%$ of the proteins identified in the studies of Kim et al ${ }^{6}$ and Becky et al. ${ }^{7}$, respectively. Additional $979(11.4 \%)$ proteins were identified by brain proteome analysis for the first time (Figure 1B and Supplementary Table 1 A-B). Thus, we generated a broad and high-coverage proteomic dataset with identification depth similar to that achieved by the two most comprehensive datasets reported in previous studies ${ }^{6,7}$.

Then, to provide a comprehensive protein abundance analysis, the proteome abundance in BA46 was calculated by the intensity-based absolute quantification (iBAQ) method ${ }^{10}$. A total of 7,129 proteins were successfully quantified, and their 
abundances spanned 7 orders of magnitude (Supplementary Figure 3). Approximately $4 \%$ of the most abundant proteins (292 proteins) accounted for $75 \%$ of the total protein abundance and were considered high-abundance brain proteins in the present study. The remaining $20 \%$ and $5 \%$ of the total protein abundances corresponded to 1,513 mid-abundance proteins and 5,324 low-abundance proteins, respectively (Figure 1C, Supplementary Table 1C). Gene ontology (GO) analysis revealed that nervous system structure and function were enriched in this brain proteome, especially in the case of high-abundance proteins (Figure 1D, Supplementary Table 1D), implying the important role of high-abundance proteins in brain functions.

Finally, to investigate specific proteomic profiling in BAs and explore the associations between BA protein expression and function, we used iTRAQ-based quantitative proteomics to generate a region-resolved proteome map of the brain covering 29 BAs with important significance for research and clinical applications (Figure 1A and Table 1). A total of 4,308 proteins were quantified in all BAs. The batch effect of 4 batches was evaluated by qualitative and quantitative analysis. The median CV of four batches was around 6\% (Supplementary Figure 2A), and the Venn diagram of protein identification in four batches (Supplementary Figure 2B) showed high overlapping. The log ratio distributions of 29 iTRAQ quantification results(Supplementary Figure 2C) showed similar normal distribution, and the detailed ratio distributions of each BA in four batches (Supplementary Figure 2D) showed similar distributions.

A total of 1,241 proteins were characterized by interarea variability (the difference between the maximum and minimum ratios $\geq 2$, detailed in Method section). The Human Brain Proteome Atlas(www.brain-omics.com) was constructed to enhance visualization and use of these data, which are publicly accessible (Supplementary Figure 4).

\section{Brain proteomic atlas reflects functional parcellations}

Proteins are the main components and functional operators in the cells; thus, BAs 
with similar protein expression patterns may have similar cytoarchitecture or perform similar functions. Based on the similarities of protein expression patternsK-means clustering ${ }^{11}$ was used for the classification of BAs. The consensus cumulative distribution function (CDF) plot and silhouette plot (Supplementary Figure 5) based on the consensus matrix were used to group 29 BAs into 6 clusters (detailed data in Methods section, Figure 2A, and Table 1).

The cingulate cortex cluster (cluster 4) included BAs in the cingulate cortex, subgenual cingulate cortex, and entorhinal cortex. All these BAs belong to the transition areas between the allocortex (3 layers of neuronal cell bodies) and neocortex (6 layers of neuronal cell bodies). Therefore, the cytoarchitecture of the cingulate cortex cluster was different from that of the neocortex ${ }^{12}$. At the proteome level, the cingulate cortex cluster was characterized by protein patterns distinct from those of other clusters, which were mainly located in the neocortex (Figure 2A), implying that the brain proteome can reflect the differences in cytoarchitecture between the cingulate cortex and the neocortex.

The remaining 23 BAs within the neocortex were grouped into 5 clusters, and BAs within a single cluster were functionally similar. For example, the vision cluster (cluster 6) comprised a group of BAs (BAs 17, 18, 19, 37, and 34) engaged in visual processing (Figure 2B, Table 1). BAs 17, 18, and 19 are located in the occipital lobe, and BA37 is adjacent to BA19. All four BAs correspond to classic visual processing regions ${ }^{13-15}$. BA34 is located in the parahippocampal gyrus and is not spatially connected to other four BAs, although BA34 was reported to be related to visual processing ${ }^{16}$. Another example is the auditory and Broca's area cluster, which is composed of several spatially separated but functionally related BAs (Figure 2B). BA44/45 of this cluster (Broca's area and motor language area), BA8 (writing language area) ${ }^{17}$ and BA7 (engaged in speech ${ }^{18}$ ) are directly involved in language processing, whereas BA41/42 (auditory cortex) and BA26/29/30 (engaged in sound processing) ${ }^{19}$ are associated with auditory processing, which is indirectly linked to language processing ${ }^{3,8}$. 
Interestingly, two language processing BAs, BA22 (classic Wernicke's area and sensory language area) and adjacent BA21 (also considered a part of Wernicke's area ${ }^{20}$ ), were clustered into the Wernicke's area cluster. This cluster was characterized by proteomic patterns distinct from the auditory and Broca's area cluster. To illustrate the differences in protein expression between these two clusters, we compared differential proteins between the two clusters. A total of 88 and 108 proteins were expressed at higher levels in the Wernicke's area cluster and auditory and Broca's area cluster, with a 1.5-fold change, respectively (Supplementary Table 3). Differentially expressed proteins were enriched in metabolic pathways, suggesting metabolic differences between the two language regions (Supplementary Figure 6A). These proteins are also related to neurotransmitter pathways ${ }^{21}$, including glutamate, $\gamma$-aminobutyric acid (GABA), and dopamine pathways (Supplementary Figure $6 \mathrm{~B})^{21}$. In particular, the GABA receptor was expressed at a higher level in the auditory and Broca's area cluster compared to that in the Wernicke's area cluster in agreement with previous reports on an important role of the GABA receptor in the physiological functions of Broca's area. An inhibitor of the AGABA receptor causes a dysfunction in Broca's area and induce stuttering ${ }^{22}$, and a GABA agonist improves chronic Broca's aphasia ${ }^{21}$. Therefore, these differentially expressed proteins provide valuable information about the intrinsic mechanism of language processing.

\section{Cluster/BA signature proteins linked to specialized function of clusters/BAs}

BAs with similar protein expression patterns tend to have similar cytoarchitecture and functions; thus, cluster signature proteins (proteins that were expressed at a high level in a certain cluster) may provide information about specialized functions of a cluster. Iterative signature analysis (ISA, details described in the Methods section) ${ }^{23}$ was used to identify a total of 474 cluster signature proteins in the present study (Figure 3A and Supplementary Table 4A). Pathway, function, and disease annotations of these proteins are shown in Supplementary Table 4B-E, Figure 3B-3C, and 
The cingulate cortex cluster had the largest number of signature proteins, 266 out of all 474 signature proteins. These proteins included 76 (26.3\%) and 140 (52.6\%) high- and mid-abundance proteins, respectively (Figure 3D and Supplementary Figure 7C-7E), corroborating the results regarding the differences in cytoarchitecture and protein expression between the cingulate cortex cluster and the neocortex. Functional analysis showed that these signature proteins are enriched in metabolic pathways, including important enzymes for oxidative phosphorylation (NADH dehydrogenase), glycolysis (ALDOA, PGK1, PGAM1, and ENOA), glycogen turnover (TPIS), and amino acid synthesis (ASS1 and PDHX) (Figure 3E), indicating high metabolic activity of the cingulate cortex. These results are in agreement with the metabolic analysis of the cingulate cortex reported in a previous study ${ }^{24}$. A total of 21 signature proteins were involved in cognition, emotion, and memory (Supplementary Table 4F), consistent with the functional characteristics of the cingulate cortex ${ }^{24-26}$. These results are in agreement with the functional characteristics of the cingulate cortex. Disease annotations indicated that proteins related to neurological degenerative diseases (Huntington's disease, Parkinson's disease, and Alzheimer's disease) were enriched in the signature proteins of the cingulate cortex cluster. Several key proteins associated with signaling in Alzheimer's and Parkinson's diseases were expressed at high levels in the cingulate cortex cluster (Supplementary Figure 7F-7G). These proteins include tau (MAPT) and alpha-synuclein (SYUA), which are important biomarkers for Alzheimer's disease ${ }^{27,28}$ and Parkinson's disease ${ }^{29}$, respectively (Figure 3F). The pathological accumulation of these two proteins is etiologically correlated with the development of Alzheimer's ${ }^{28}$ and Parkinson's diseases ${ }^{29}$. The data of Western blot analysis showed that the protein expression patterns of MAPT and SYUA were consistent with the patterns detected by proteomic analysis (Supplementary Figure 8A-B). The results of IHC-Fr also demonstrated that MAPT and SYUA were expressed at a high level in BA25 compared to that in BA4 (Supplementary Figure 8C). The results of this study demonstrated the regional distribution of MAPT and 
SYUA for the first time, adding new information about pathogenesis and vulnerability of these two neurodegenerative diseases in the cingulate cortex. Furthermore, another brain (brain 2) was used to validate the cluster-specific signature proteins by parallel reaction monitoring (PRM). Detailed clinical information on brain 2 is provided in the Methods section. PRM analysis validated that 28 signature proteins were expressed at a high level in cingulate cortex cluster (Supplementary Table 5A-5B).

The motor and sensory cluster had 99 signature proteins. These proteins were enriched in the protein kinase A (PKA) pathway (Figure 3B). The PKA pathway is associated with brain motor function (neuronal plasticity in motor neurons ${ }^{30,31}$ ) and sensation (pain sensitization in sensory neurons ${ }^{32,33}$ ). Striatal-enriched protein tyrosine phosphatase (STEP), a molecule downstream of PKA, is associated with motor skill learning in the motor cortex ${ }^{34}$. Other signature proteins with potentially interesting functions include the neuron survival protein EMP2Aandmitochondrial 28S ribosomal protein S34(MRPS34) (Figure 3F). The functional loss of EMP2A was reported to cause sensorimotor cortex excitability in Lafora disease ${ }^{35}$. MRPS34 mutation causes delayed psychomotor development and neurodevelopmental deterioration in Leigh syndrome ${ }^{36}$. Immunological analysis confirmed that EMP2A and MRPS34 were expressed at a high level in the motor and sensory cluster (Supplementary Figure 8). Two signature proteins of motor and sensory cluster, EMP2A and PTN5, were validated by PRM (Supplementary Table 5A-5B).

The vision cluster included 49 signature proteins enriched in the methyl-CpG-binding protein 2 (MECP2)-regulated transcription factor pathway. MECP2 and transcriptionally regulated transcription factors are involved in neurological system and neuropsychiatric disorders ${ }^{37}$ (Figure 3B). MECP2 plays an important role in normal development of the central nervous system and maintenance 270 of neurological functions. Mutation of the MECP2 gene leads to Rett syndrome, which is an autism spectrum-associated disorder with visual impairment ${ }^{38}$. In a mouse 272 model, knockout of MECP2 causes progressive dysfunction of the visual cortex and 
TAGLN, and CAVN1, were confirmed to be expressed at high level by PRM (Supplementary Fig 10B, Supplementary Table 5A-5B). Additionally, Becky et al ${ }^{7}$ reported a proteomic comparison of BA17 (primary visual cortex) and BA46 (DFC)in 11 individuals ranging from early infancy to adulthood.By comparing the 49 vision cluster signature proteins in present study with Becky's study, 16 were expressed at high level in primary visual cortex in both studies (Supplementary Table 5E).

Additionally, we focused on BA signature proteins (protein abundance in one or two BAs that is more than twofold higher than the median abundance) to investigate BA-specific functions. A total of $134 \mathrm{BA}$ signature proteins were identified based on the analog criteria suggested by Uhlenet al ${ }^{40}$ (details are provided in the Methods section), (Figure 4A and Supplementary Table 4G). These BA signature proteins included several proteins closely associated with the corresponding brain functions. In the cingulate cortex, superoxide dismutase [Cu-Zn] (SOD1) was identified as a signature protein in the anterior cingulate cortex (BA32 and BA24). SOD1 mutation interferes with GABAergic neurotransmission, leading to cortical neuronal loss in this brain area $^{41}$. In the neocortex, FAM107B was identified as a signature protein in BA41/42 (auditory cortex) (Figure 4B). Knockout of FAM107B leads to hearing impairment in mice ${ }^{42}$. Three BA signature proteins, transgelin-2 (BA17 and BA18), RAD23A (BA40), and FAM107B (BA41/42), were validated by Western blot (Figure 4B, Supplementary Figure 9). Five BA signature proteins, NEFH (BA4), PMP2 (BA4), CALB2 (BA28), TAGLN (BA17 and BA18), and FAM107B (BA41/42), were validated to be expressed at a high level in corresponding BAs in brain 2 (Supplementary Table 5C-5D). Two primary visual cortex signature proteins, TAGLN and H1F0 were also found to be expressed at high level in primary visual cortex in Becky's study ${ }^{7}$ (Supplementary Table 5E).

Overall, these findings demonstrated intrinsic links between specialized functions of the brain clusters and BAs and molecular functions of the corresponding signature proteins, which may help to understand the mechanisms of physiological or pathophysiological functions of the brain. 


\section{Molecular annotation of uncanonical functions of BAs}

304

305

306

307

308

309

310

311

312

313

314

315

316

317

318

319

320

321

322

\section{Functional connection between the motor cortex and anterior cingulate cortex}

The anterior cingulate cortex is tightly connected to the motor cortex through neuronal cell projections according to the results of fluorescence labeling imaging in rats $^{43}$. In humans, Caruanaet al. demonstrated that the anterior cingulate cortex plays important roles in the control of complex motor behaviors according to the results of intracorticalelectrical stimulation ${ }^{44}$. Molecular evidence of the connection between these two functional brain regions requires additional investigation.

Proteomic data obtained in the present study demonstrated that a series of proteins were expressed at a high level in the primary motor cortex (BA4) and anterior cingulate cortex (BA33) (Figure 5A, Supplementary Table 6A). Most of these proteins are associated with motor neuron structure and function (Figure 5B). Neurofilament proteins (NEFL, NEFM, NEFH, and INA) are the main components of motor neurons ${ }^{45}$ and are necessary for the maintenance of the number, morphology, viability, and regenerative capability of motor neurons ${ }^{46-48}$. Myelin basic protein promotes myelination of motor neurons ${ }^{49}$. MIF ${ }^{50}$ and PVALB ${ }^{51}$ are associated with amyotrophic lateral sclerosis, a neurodegenerative disease targeting motor neurons ${ }^{52}$. Thus, these proteins may represent molecular basis of the functional connections between the primary motor cortex (BA4) and anterior cingulate cortex (BA33) and may also play a role in the motor functions of the anterior cingulate cortex.

\section{The motor and sensory cluster may be involved in anxiety}

Anxiety disorder is a disease characterized by excessive and persistent worry and fear and is very common worldwide (lifetime prevalence is $5 \sim 25 \%$ of the population), representing a substantial economic burden ${ }^{53,54}$. The sensorimotor cortex has been reported to be associated with social anxiety disorder ${ }^{55}$ and anxiety-related personality traits ${ }^{56}$ according to the results of electrophysiological studies. However, the molecular basis of anxiety in the sensorimotor cortex requires further investigation. 
Proteomic data obtained in the present study indicated that 30 of the 1,241 proteins with inter area variability are involved in anxiety or anxiety-like behaviors according to IPA (Supplementary Table 6B). Clustering analysis indicated that nearly half $(14,46.6 \%)$ of these 30 proteins were expressed at a high level in the motor and sensory cluster (Figure 5C). Some of these 14 proteins, including ADCY8, GRIA1, GRIN2A, PLcB4, SLITRK5, NOS1, and GRM2, formed a protein interaction and regulation network and participated in the expression of anxiety and anxiety-like behaviors (Figure 5D). PlcB4 ${ }^{57}$ and NOS1 ${ }^{58}$ were expressed at a high level in the medial septum and the hippocampus (two anxiety behavior-related regions), respectively, and inhibition of these genes in the regions with high levels of expression increased anxiolytic effects in a mouse model. GRIN2A and GRIA1 are two important glutamate receptors involved in the function of the sensorimotor cortex 59 and also involved in anxiety. Knockout of GRIN2A ${ }^{60}$ and GRIA1 ${ }^{61}$ causes a decrease in anxiety in a mouse model. Thus, we hypothesized that these proteins are expressed at a high level in the motor and sensory cluster and may be involved in anxiety-related functions in the sensorimotor cortex, which may explain the molecular basis of anxiety-related processes in the sensorimotor cortex.

\section{Transcriptomic characterization of brain BAs}

The transcriptomes of brain BAs were explored to reveal the characteristics of the brain functions. 29 BAs by RNA-seq were analyzed, and 21 samples passed the quality control $(\mathrm{RIN}>=6,28 \mathrm{~S} / 18 \mathrm{~S}>=0.7$, gene mapping $>=40 \%)$ and were used for further functional analysis. An average of 17,137 transcripts was detected based on the RNA-seq data (Supplementary Table 7A, Supplementary Figure 10). Comparison of the brain proteome library and the transcriptomic database of BA46 identified 8,411 transcriptomic entries $(97.8 \%$ ) for 8,600 proteins detected by LC-MS (Figure $6 \mathrm{~A})$. The proteomic data covered $48.8 \%$ of the transcriptomic data, covering $61 \%$ expression level>10 RPKM (Figure 6B). 
A total of 17,091 genes were quantified in 21 BAs. PCA of the transcriptomic data showed that BA4 (primary motor cortex) was different from other BAs in agreement with the finding of the Allen Institute that a series of genes are specifically enriched in the primary motor cortex ${ }^{1}$. The cingulate cortex cluster tended to be partially separated from other BAs, which was partially consistent with the proteomic results. However, four functionally distinct clusters distinguished by proteomic analysis could not be found based on the transcriptomic data (Figure 6C).

The BA signature genes at the transcriptomic level were used to explore BA-specific functions. A total of 1,319 BA signature genes were identified (Figure 6D and Supplementary Table 7B). Functional analysis indicated that BA signature genes were closely involved in the functions of corresponding BA. In cingulate cortex,a total of 13 signature genes of BA 28 were involved in memory function, in agreement with the functional characteristics of the ventral entorhinal cortex (BA28) ${ }^{26}$ (Supplementary Table 7C). In neocortex, motor neuron related proteins (NEFH, NEFM) ${ }^{46,47}$ were identified as signature genes of primary motor cortex (BA4), and were also expressed at high level in BA4 in our proteomic data. In Auditory cortex(BA41/42), SMPX was identified as a signature gene. Previous study reported that mutation of SMPX was associated with X-linked deafness type $4{ }^{62}$.

A total of 4,250 genes were quantified based on the data of transcriptomic and proteomic results in 21 BAs (Supplementary Table 7D). Gene products for all region pairs were classified based on fold-change similarity between mRNA and protein (details described in the Methods section). An average of $39.5 \%$ of the genes were classified into "no change" ( $\leq 2$-fold for both protein and RNA) category, and $17.4 \%$ of the genes were classified into "agree" category (same direction of changes (>2-fold) and same magnitude of fold changes) or "partially agree" category (same direction of changes (> 2-fold) and different magnitude of fold changes) category. In addition, $20.6 \%$ or $15.7 \%$ of the genes were considered "protein only" ( $\geq 2$-fold change in protein level only) or "RNA only" ( $\geq 2$-fold change in mRNA level only), respectively. 
387 In contrast, $6.7 \%$ of the genes corresponded to opposite differences in the mRNA and 388 protein levels (> 2-fold) (“disagree” group) (Figure 6E).

389 To show the differences between transcriptome and proteome on brain functions, 390 BA4 (Primary motor cortex) and BA46 (DFC) were compared and the scatter plots of 391 the mRNA and protein fold change between them were shown in Figure 6F 392 (Supplementary Table 7E, other BA pair comparison data in Supplementary Figure 393 11). According to IPA functional annotation of the differential gene products between 394 BA4 and BA46, Progressive motor neuropathy and Parkinsonism related genes were 395 enriched in "protein only" and "partially agree" categories, which could reflect the 396 characteristics of the Primary motor cortex. In contrast, the cognitive impairment and 397 the depressive disorder related genes showed a bias toward"mRNA only" categories, 398 reflecting the characteristics of the DFC Additionally, the neurodevelopment related 399 genes were enriched in the "agree" category, consistent with the Becky's findings 7 400 (Figure 6G,Supplementary Table 7F). 
402

403

404

405

406

407

408

409

410

411

412

413

414

415

416

417

418

419

420

421

422

423

424

425

426

427

428

\section{Discussion}

In this study, we constructed a Chinese cerebral cortex proteomic atlas of 29 BAs from a donor without neurological diseases. Similarities in the protein expression patterns indicated that these 29 BAs can be grouped into six clusters. The cluster- and BA signature proteins can provide molecular evidence for annotation of cytoarchitecture, function, and disease vulnerability of these clusters or the corresponding BAs. All data are publicly available in the Human Brain Proteome Atlas (HBPA) (www.brain-omics.com).

In this brain proteome atlas, BAs with similar protein expression patterns have similar functions, even though they are not spatially adjacent, which reflects functional parcellations. BAs within the cingulate cortex cluster and vision cluster are characterized by both spatial proximity and functional relevance. However, BAs within the auditory and Broca's area cluster are not directly adjacent and are functionally connected (speech and auditory). These results indicated that the proteomic map mainly reflects the functional parcellations of the human cerebral cortex. Furthermore, proteomic similarity of BAs may provide molecular annotations for functional connections between BAs. For example, BA38 (temporal pole) is an enigmatic region with largely unknown function ${ }^{63}$. The brain connectome indicated a functional connection between BA3/1/2-BA38 and BA21/22-BA38 ${ }^{3}$. The results of fMRI experiments indicated that BA38 is involved in speech comprehension and considered a part of "extended Wernicke's area" ${ }^{64,65}$. A study on Braille reading in blind individuals using brain imaging methods indicated thatBA38 can integratesensory-motor and language comprehension. ${ }^{63}$ In the present study, BA38 was characterized by protein expression patterns similar to both the sensorimotor cortex (BA3/1/2, BA4, and BA6) in the motor and sensory cluster and Wernicke's area (BA21 and BA22). We suggest that BA38 may play integratory roles in both sensory-motor functions and language comprehension. 
Additionally, the present study identified 474 cluster- and 134 BA signature proteins. These proteins reveal intrinsic links between their molecular functions and specialized functions of the brain regions. The cingulate cortex contains an extremely large number of signature proteins compared to that of other neocortex regions. These signature proteins were enriched in metabolic pathways, mainly glycolysis, glycogen turnover, and amino acid synthesis.Previous studies demonstrated that astrocytes had unique metabolic pathways of brain glucose metabolism compared to those in neurons 66. First, glycolysis is predominant in astrocyte glucose metabolism. Second, the storage and turnover of glycogen are mainly concentrated in astrocytes. Third, TCA cycle-derived amino acids, such as aspartate (Asp), glutamate (Glu), glutamine (Gln), arginine (Arg), and $\gamma$-aminobutyric acid (GABA), are synthesized in astrocytes using astrocyte-specific enzymes ${ }^{66}$. The combination of previous reports and proteomic data obtained in the present study indicates the activation of astrocyte metabolism in the cingulate cortex, which may correspond to metabolic characteristics of the cingulate cortex. Additionally, the results of the present study suggest that some signature proteins in the cingulate cortex cluster participate in the pathogenesis of some neurological diseases, such as MAPT in Alzheimer's disease and SYUA in Parkinson's disease. Previous studies have shown that the accumulation of these two proteins in the cingulate cortex is a pathological feature of the corresponding diseases 28,29. Abnormalities in the cingulate cortex play a central role in the development of Alzheimer's ${ }^{24}$ and Parkinson's diseases ${ }^{67,68}$. These signature proteins may be investigated to determine molecular mechanisms of neurological diseases, especially in some specific brain regions. Thus, information about these proteins may substantially improve our understanding of the molecular basis of brain functions and pathogenesis.

Furthermore, the data of the present study provide molecular annotations of the noncanonical functions of brain regions. Molecular function analysis of these data provides molecular basis for functional connections between the anterior cingulate cortex and motor cortex and may explain the molecular basis of anxiety in the 
sensorimotor cortex. These findings promote our understanding of the molecular mechanisms of the noncanonical functions of brain regions. Therefore, proteomic data obtained in the present study may be used to investigate new functions of BAs or new functional connections between BAs.

In present study,the comparison of BA transcriptome and proteome indicated thatthe proteomic data could separate the cerebral cortex into 6 clusters, which were associated with BA's function. However, transcriptomic data did not show similar classification, which was consistent with Allen results ${ }^{1}$. Above results indicated that brain proteomic data might preferably reflect BA function. Functional analysis of the trancriptome suggested that it could also reflect the function characteristics of BAs. $17.4 \%$ gene showed similar differential expression trend as the protein (such as neurodevelopment), and some BA signature genes (NEFH, NEFL in primary motor cortex) also showed consistent expression patterns as the protein. Moreover, $15.7 \%$ gene only differentially expressed in mRNA level, and some of which were associated with BA function (cognition impairment, depressive disorder in DFC). Above data demonstrated that transcriptome and proteome showed different characteristics of brain functions. According to previous studies ${ }^{7}$, in neuron mRNA and proteins might locate in different brain regions. Synapse proteins were transmitted from the nucleus into another BA through axon, so they could be detected in both BAs. However, mRNA was limited to the source BA containing cell body, so they were only identified in source BA. Thus, such genes would be differentially expressed only on mRNA level. Above study could partially account for the differences between transcriptome and proteome.

This study established a Chinese proteome atlas of the brain; however, the following issues need to be resolved in the future studies. First, we drafted a global multiregional proteomic map of the left hemisphere of a single brain. Previous proteomic and transcriptomic studies suggested that individual variations in age and gender were substantially lower than variations across brain regions ${ }^{1,7}$. Proteomic 
486 atlas generated in the present study can represent common characteristics of the

487 normal human cerebral cortex to some extent; however, future studies should consider

488 inclusion of multiple individuals balanced with regard to gender, age, and brain 489 hemisphere sampling and should use more sensitive and accurate proteomic 490 technologies. Second, this pilot atlas includes only 29 BAs with important brain 491 functions, and future studies should include additional important regions, such as the 492 cerebellar cortex, hippocampus, and other important nerve nuclei, to generate a more 493 comprehensive atlas. 


\section{Tissue procurement.}

497

Samples from frozen postmortem human brain specimens were obtained following the SOP of the Human Brain Bank of China facilitated by the China Human Brain Bank Consortium (see Qiuet al. ${ }^{9}$ for details on sample collection, handling, and preservation). Basic demographic information, medical information, and medical records of the donor were obtained. Brain 1 specimens were collected from a 54-year-old willed male donor who died of lung cancer and did not have any history of brain metastasis, trauma, or other diseases. Brain 2 was collected from a 93-year-old willed female donor who died of heart failure and did not have neurological diseases. The scalp was incised along the coronal plane from the ear to top of the skull and turned over to expose the skull. The skull was cut apart at the sagittal suture starting $1 \mathrm{~cm}$ above the eyebrows and continuing to the occipital protuberance, and the dura was removed. The cerebrum, brain stem,cerebellum, and part of the cervical cord were completely removed and temporarily preserved in cold saline. Twenty-nine samples of the cortex from various BAs were dissected from the left cerebral hemisphere and stored at $-80^{\circ} \mathrm{C}$ for protein extraction. Routine hematoxylin/eosin, silver, and immunohistochemical staining procedures for amyloid-beta, p-tau, and alpha-synuclein were performed in the visual cortex, inferior parietal lobule, superior and middle temporal gyri, medial frontal cortex, amygdala, anterior cingulate gyrus, hippocampus, basal ganglia, cerebellum, middle brain, cerebral pons, and medulla in the right cerebral hemisphere of the brain sample; the data were used for pathological diagnosis to exclude neurodegenerative diseases.According to the Standardized Operational Protocol ${ }^{9}$, we performed HE staining of the brain areas, including BA40, 24, 17, 41/42/22, to confirm that brain is cancer-free.

\section{Ethical statements}

Brain tissue was collected after obtaining consent from the donor and the next of 
523 kin using a signed informed consent form and the project was approved bythe 524 Institutional Review Board of the Institute of Basic Medical Sciences, Chinese 525 Academy of Medical Sciences (approval number: 009-2014).

\section{Sample preparation}

527 A total of 29 brain tissue samples were selected for proteomic analysis $(80 \mathrm{mg}$ 528 each). The samples were rinsed with PBS until washing fluid was clear, and each 529 sample was lysed with lysis buffer (containing $8 \mathrm{M}$ urea,2.5 M thiourea,4\% CHAPS, $53065 \mathrm{mM}$ DTT, and 50mMTris-HCl) in a homogenizer on ice. The lysate was 531 centrifuged at $14,000 \times \mathrm{rpm}$ at $4^{\circ} \mathrm{C}$ for $20 \mathrm{~min}$, and the supernatant was collected. The Bradford method was used to measure the protein concentration. Proteins were 533 digested by the FASP method, as previously described ${ }^{69}$.The peptides were extracted by an Oasis C18 extraction column and quantified by the BCA method.

\section{iTRAQ labeling and 1D off-line separation}

Digested peptides were labeled with 8-plex iTRAQ reagent according to the manufacturer's protocol (Sciex, Framingham, MA). The 28 Brodmann areas were randomly divided into 4 groups (Supplementary Table2) and labeled byiTRAQ8-plex agent.In each group, BA46 was used as common internal reference sample.The labeled samples within each experiment were mixed together. To reduce sample complexity, the peptide samples were fractionated through a high-pH RPLC system.

542 The samples were reconstituted in $100 \mu \mathrm{L}$ of buffer $\mathrm{A}(1 \mathrm{mM}$ ammonia, $\mathrm{pH}=10)$ and 543 loaded onto a column (4.6 mm×250 mm, Xbridge C18, $3 \mu \mathrm{m}$,Waters,Milford, MA).

544 The elution gradient was composed of 5-25\% buffer B (90\% ACN, 1 mM ammonia, $545 \mathrm{pH}=10$ ) ataflow rate of $0.8 \mathrm{~mL} / \mathrm{min}$ for $60 \mathrm{~min} .60$ fractions were collected, dried, 546 andresuspended in $0.1 \%$ formic acid; the fraction were pooled into 20 samples using a 547 stepwise concatenation strategy to combine every $20^{\text {th }}$ fraction $(1,21,41 ; 2,22,42 ; \ldots)$. 548 A total of 100 fractions from a group of unlabeled samples and four groups of 549 iTRAQ-labeled samples and were further analyzed by LC-MS/MS. 


\section{LC-MS/MS analysis}

The pooled mixtures of iTRAQ-labeled samples were analyzed using a self-packed RP C18 capillary column $(75 \mu \mathrm{m} \times 100 \mathrm{~mm}, 1.9 \mu \mathrm{m})$. The elution gradient was composed of 5-30\% buffer B (0.1\% formic acid and $99.9 \% \mathrm{ACN})$ at a flow rate of $0.3 \mu \mathrm{L} / \mathrm{min}$ for $45 \mathrm{~min}$. An LTQ Orbitrap Fusion Lumos instrument (Thermo Fisher Scientific, Waltham, MA) was used to acquire raw data. The following parameters were used for MS data acquisition: top speed data-dependent mode (3 s) was used for full scan; full scans were acquired in an Orbitrap at a resolution of 60,000; the MS/MS scans were acquiredat a resolution of 15,000 with charge state screening (excluding precursors with unknown charge state or +1 charge state); $38 \%$ normalized collision energy in HCD mode; 30s dynamic exclusion (exclusion size list 500);1.6 Da isolation window. Each fraction from the iTRAQ-labeled sample was run twice. For label-free analysis, 32\% normalized collision energy in HCD was used, other parameters were identical to the iTRAQ. Each fraction from the unlabeled sample was run three times.

\section{Database search}

The MS/MS spectra were searched by Proteome Discoverer software (v3.1, Thermo Fisher Scientific) using the SwissProt human database downloaded from the UniProt website (www.UniProt.org). Trypsin was selected as the cleavage specificity parameter, andallowed a maximum of two missed cleavagesites. For iTRAQ quantification, carbamidomethylation of cysteine and iTRAQ 8-plex labels were set as fixed modifications, and the oxidation of methionine, deamidation of asparagine and glutamine, and carbamylation of lysine and peptide $\mathrm{N}$-terminus were used as dynamic modifications. The searches were performed using a peptide tolerance of 10 ppm and a production tolerance of $0.02 \mathrm{Da}$. A $1 \%$ false-positive rate at the protein level was set as a filter, and each protein had to contain at least 1 unique peptide. For label-free quantification, carbamidomethylation of cysteine was used as a fixed modification, and other search parameters were identical to the parameters used for 
578 iTRAQ quantification. The abundance of each protein was estimated by Progenesis 579 software (Nonlinear Dynamics, version 4.0), and the iBAQ value of each protein was 580 calculated based on the abundance of a protein divided by the theoretical polypeptide 581 number of the corresponding protein.

Each iTRAQ experiment contained seven BA samples and a common internal reference sample (BA46). In each experiment, the abundance ratios of the seven samples in BA46 were calculated, and the average protein abundance ratios of two technical replicates were used for further analysis. To ensure reliability of the data, the missing values in any two technical replicates of any group were excluded from subsequent analysis. The abundance ratios of proteins identified in all four experiments were merged into a single table in which each row represented a protein and each column represented a BA. In the column for BA46, all protein ratios were filled with 1 . Then, the ratios were $\log 2$ transformed and normalized to a column median of 0 and a row median of 0 . Thus, the positive value was higher than median, and the negative value were lower than median. Through row-wise normalization, the expression pattern of each row/protein in all the samples could be better exhibited, and it would be helpful for following cluster analysis and signature proteins filtering.

For better cluster analysis and signature protein selection the proteins without inter-area variability were filtered. The fold changes of all proteins between all BA pairs were calculated. The distribution of $\log 2$ fold change showed that the and Q3 + 1.5 interquantile range was 1.57. A strict filter (fold change $>2$ ) was used as the threshold of outliers, which would be helpful to signature proteins. To identify the differential proteins between Wernicke's area cluster and auditory and Broca's area cluster, a relative relax threshold (1.5 fold change) was used to find more differential proteins, which would be helpful for pathway or function enrichment analysis. 
606 Euclidean distance was performed 1,000 times on the subsampled data. The optimal

607 number of clusters was selected using the consensus matrix, consensus cumulative 608 distribution function (CDF) plot, and silhouette plot. The consensus matrix showed 609 the best clustering results at $\mathrm{k}=3$. However, the CDF of consensus increased at 610 greater $\mathrm{k}$ values. At $\mathrm{k}=6$, a relative increase in CDF reached a very small value. The 611 silhouette plot at $\mathrm{k}=6$ showed a mean silhouette score of 0.72 , and all scores were 612 positive, indicating that clusters were well separated. Thus, BAs were clustered into 613 six clusters.

614 Cluster signature proteins were identified by a biclustering algorithm known as 615 iterative signature algorithm (ISA). ISA was implemented using the isa2 package for $616 \mathrm{R}^{23}$. Biclustering was restricted to upregulated modules in both rows and columns 617 with a score threshold of 0.7 . For all six clusters, modules that included at least $80 \%$ 618 BAs inside a cluster and no BAs outside of the cluster were selected. Proteins in the 619 selected modules with scores that passed the threshold of 0.7 were considered 620 signature proteins for this cluster.

621 An adequate cutoff ratio was determined to identify proteins overexpressed in 622 specific BAs. The differences in the $\log 2$ protein abundance ratios between all BA 623 pairs for all proteins were calculated, and the data were normally distributed with a 624 mean of 0.00 and a standard deviation (sd) of 0.35 . The $\log 2$-fold change cutoff was 625 then determined to be 1 , which approximated the mean $+3 *$ sd. Thus, an area 626 signature protein was defined as a protein with abundance level in one or two 627 particular BAs of at least twofold of the median abundance level in all 29 BAs.

\section{Downstream Bioinformatics analysis}

All proteins of interest were analyzed using the David database (http://david.ncifcrf.gov/) and compared with the whole human genome for GO enrichment analysis. For IPA analysis, the signature or differential proteins were

632 evaluated using IPA software (Ingenuity Systems, Mountain View, CA), and the enrichment of the disease, function, and canonical pathway categories were analyzed 
634 according to the P-value rankings. For reactome analysis, cluster signature proteins

635 were mapped to the reactome database in the pathway category according to the 636 P-value rankings. For KEGG analysis, cluster signature proteins were mapped to the

637 KEGG database in the pathway category according to the P-value rankings.

638

639

640

641

642

643

644

645

646

647

648

649

650

651

652

653

654

655

656

657

658

659

660

661

\section{IHC and Western blot}

Western blot analysis of brain samples was performed to validate the proteomic quantitation of several selected candidate proteins (tau, alpha-synuclein, EPM2A, MRPS34, TAGLN, FAM107B, and RAD23A). Proteins extracted from the brain tissues were separated by SDS-PAGE and electro-transferred to a PVDF membrane (Millipore, Bedford, MA). The membrane was blocked with 2\% (v/v) BSA for $2 \mathrm{~h}$ at room temperature and incubated with primary antibodies and a peroxidase-conjugated secondary antibody. The membranes were visualized using Immobilon Western chemiluminescent horseradish peroxidase substrate (Millipore), and the bands were analyzed by ImageJ software.

Formalin-fixed, paraffin-embedded brain tissue samples were used for IHC-Fr analysis. The tissue sections were deparaffinized and rehydrated in xylene and a graded ethanol series, and antigen retrieval was performed in a pressure cooker by boiling in sodium citrate buffer ( $\mathrm{pH}$ 6.0) for $2 \mathrm{~min}$. Then, endogenous peroxidases were blocked with $0.3 \% \mathrm{H}_{2} \mathrm{O}_{2}$ for $15 \mathrm{~min}$, and the samples were incubated in the presence of $2 \%$ fetal calf serum for $20 \mathrm{~min}$. Primary antibodies were incubated at $4{ }^{\circ} \mathrm{C}$ overnight, and peroxidase-labeled polymer conjugated to anti-mouse, anti-rabbit, or anti-goat immunoglobulins was incubated for $1 \mathrm{~h}$. The sections were counterstained with Mayer's hematoxylin and dehydrated, and the images were acquired under a microscope.

Primary antibodies for Western blot and IHC-Fr against MAPT (rabbit monoclonal, ab76128), SYUA (rabbit monoclonal, ab138501), EPM2A (rabbit monoclonal, ab129110), MRPS34 (mouse monoclonal, HPA042112, Sigma), TAGLN (rabbit polyclonal, ab14106), and RAD23A (mouse monoclonal, ab55725) were 
662 purchased from Abcam (Cambridge, UK). Antibodies against FAM107B (rabbit 663 polyclonal, ab122566) were purchased from Sigma-Aldrich (St Louis, MO).

\section{PRM analysis}

665 Selected cluster and BA signature proteins were validated in 27 BAs of brain 2 by 666 PRM. To estimate the quality of the data, we used the mixed sample as quality control 667 (QC) during the analysis, before and after all samples, and every 7-8 samples. Two 668 technical repeats of each sample were assayed. The average Pearson correlation 669 coefficient of QC samples was 0.97, indicating the reproducibility of the QC.

The samples were analyzed on a C18 RP self-packed capillary LC column (75 $\mu \mathrm{m} \times 100 \mathrm{~mm})$. The elution gradient was composed of $5-30 \%$ buffer B $(0.1 \%$ formic 672 acid, 99.9\% ACN) with a flow rate of $0.5 \mu \mathrm{L} / \mathrm{min}$ for $45 \mathrm{~min}$. An LTQ Orbitrap Fusion Lumos instrument was used to acquire raw data. Following parameters was used for MS data acquisition: PRM mode; full scans and MS/MS scans were acquired in Orbitrap at a resolution of 60,000 and 15,000, respectively; 32\% normalized collision energy in HCD mode; 30s dynamic exclusion; The isolation window was 4, and the schedule mode window was 7 min.

PRM data processing was performed by Skyline 19.1 software as previous described ${ }^{69}$. The peptide abundance was normalized to TIC in each sample,which was extracted by Progenesis QIP software (Waters).

RNA seq and data processing

RNA-seq was performed by BGI cooperation (Shenzhen, China). For transcriptome data analysis, read counts were normalized using the median of ratios normalization method in DESeq2 to eliminate the variations in sequencing depth and RNA composition in the samples ${ }^{70}$. Normalized counts were logarithmically transformed with base 2. A total of 17,091 transcripts with read counts $>1$ in at least $68750 \%$ of the samples were retained for downstream analysis. Transcriptomic and 688 proteomic data were matched by gene symbols. 


\section{Comparison between the proteome and transcriptome}

690 The genes commonly quantified at the mRNA and protein levels were quantitatively

691 compared. Both transcriptomic and proteomic datasets were $\log 2$ transformed by

692 zero-mean normalization. The gene products for all possible region pairs were

693 classified based on their fold-change similarity between mRNA and protein. Genes

694 with the same direction of the changes at the RNA and protein levels and with the

695 same magnitude of fold change (the variability in protein level between BAs was <

696 2-fold of that at the mRNA level, assigned to the "agree" category); the genes with the

697 same direction of change but with different magnitude of fold change (larger than

698 2-fold) at the RNA and protein levels were assigned to the "partially agree" category.

699 The genes that had no significant inter regional changes in either RNA or protein

700 levels (within 2-fold) between BAs were assigned to the "no change" category. The

701 genes that had variable levels of corresponding proteins between various BAs (larger

702 than 2-fold) but did not vary at the mRNA level (within 2-fold) were assigned to the

703 "protein only" category. The genes that had variable mRNA levels (larger than 2-fold)

704 but did not vary at the protein level (within 2-fold) were defined as "mRNA" only.

705 The genes that showed opposite changes in mRNA and protein levels were assigned

706 to the "disagree" category. 
708

709

710

711

712

713

714

715

716

717

718

719

720

721

722

723

724

725

726

727

728

729

730

731

732

733

\section{CRediT author statement}

Zhengguang Guo: formal analysis, funding acquisition, validation, writing - original draft. Chen Shao: methodology, formal analysis, software visualization, funding acquisition, and writing - review \& editing. Yang Zhang: conceptualization, writing review \&editing. Wenying Qiu: resources, investigation. Wenting Li: resources. Weimin Zhu: project administration. Qian Yang: validation. Yin Huang: software.Lili Pan: visualization. Yuepan Dong: visualization. Handan Sun: investigation. Xiaoping Xiao: investigation. Wei Sun: investigation, project administration, funding acquisition, writing - review \& editing. Chao Ma: project administration, funding acquisition. Liwei Zhang: conceptualization, project administration, funding acquisition, supervision. All authors read and approved the final manuscript.

\section{Authors' ORCID IDs}

Zhengguang Guo: 0000-0002-5983-7759

Chen Shao: 0000-0002-6899-5353

Yang Zhang: 0000-0002-0033-660X

Wenying Qiu: 0000-0003-4906-8746

Wenting Li: 0000-0002-1074-0956

Weimin Zhu: 0000-0002-2420-0731

Qian Yang: 0000-0001-7159-9389

Yin Huang: 0000-0001-9289-2111

Lili Pan: 0000-0003-0258-5367

Yuepan Dong: 0000-0002-4546-3530

Haidan Sun: 0000-0003-1169-0329

Xiaoping Xiao: 0000-0001-5021-9906

Wei Sun: 0000-0002-1473-7412

Chao Ma: 0000-0003-1346-6961 
bioRxiv preprint doi: https://doi.org/10.1101/2021.03.07.434254; this version posted March 7, 2021. The copyright holder for this preprint (which was not certified by peer review) is the author/funder. All rights reserved. No reuse allowed without permission.

734 Liwei Zhang: 0000-0003-1919-3765

735 
bioRxiv preprint doi: https://doi.org/10.1101/2021.03.07.434254; this version posted March 7, 2021. The copyright holder for this preprint (which was not certified by peer review) is the author/funder. All rights reserved. No reuse allowed without permission.

\section{Competing interests}

737 The authors declare that they have no competing interests. 


\section{Acknowledgments}

740 We are grateful to Professor BaiLu, and Dr. He You from Tsinghua University for

741 helpful discussions. This work was supported by National Key Research and

742 Development Program of China (No. 2016 YFC 1306300, 2018YFC0910202),

743 National Natural Science Foundation of China (No. 30970650, 31200614, 31400669,

744 81371515, 81170665, 81560121), Beijing Medical Research (No.2018-7), Beijing

745 Natural Science Foundation (No. 7173264, 7172076), Beijing cooperative

746 construction project (No. 110651103), Beijing Science Program for the Top Young

747 (No. 2015000021223TD04), Beijing Normal University (No. 11100704), Peking

748 Union Medical College Hospital (No. 2016-2.27), CAMS Innovation Fund for

749 Medical Sciences (2017-I2M-1-009), the CAMS special basic research fund for

750 central public research institutes (No. 2017PT310004), and Biologic Medicine

751 Information Center of China, National Scientific Data Sharing Platform for

752 Population and Health. 


\section{Reference}

7551 Hawrylycz, M. J. et al. An anatomically comprehensive atlas of the adult human brain 756 transcriptome. Nature489, 391-399, doi:10.1038/nature11405 (2012).

7572 Brodmann, K. Vergleichende Lokalisationslehre der Grosshirnrinde. Leipzig : Barth, 1909 758 (1909).

7593 Fan, L. et al. The Human Brainnetome Atlas: A New Brain Atlas Based on Connectional $760 \quad$ Architecture. Cereb Cortex26, 3508-3526, doi:10.1093/cercor/bhw157 (2016).

7614 Sharma, K. et al. Cell type- and brain region-resolved mouse brain proteome. Nat Neurosci18, 762 1819-1831, doi:10.1038/nn.4160 (2015).

7635 Jung, S. Y. et al. An Anatomically Resolved Mouse Brain Proteome Reveals Parkinson 764 Disease-relevant Pathways. Mol Cell Proteomics16, 581-593, doi:10.1074/mcp.M116.061440 (2017).

7666 Kim, M. S. et al. A draft map of the human proteome. Nature509, 575-581, doi:10.1038/nature13302 (2014).

Carlyle, B. C. et al. A multiregional proteomic survey of the postnatal human brain. Nat Neurosci20, 1787-1795, doi:10.1038/s41593-017-0011-2 (2017).

Patel, R. S., Bowman, F. D. \& Rilling, J. K. Determining hierarchical functional networks from auditory stimuli fMRI. Hum Brain Mapp27, 462-470, doi:10.1002/hbm.20245 (2006). Bul/35, 270-276, doi:10.1007/s12264-018-0306-7 (2019).

Mann, K. \& Edsinger, E. The Lottia gigantea shell matrix proteome: re-analysis including MaxQuant iBAQ quantitation and phosphoproteome analysis. Proteome Sci12, 28, doi:10.1186/1477-5956-12-28 (2014).

Wilkerson, M. D. \& Hayes, D. N. ConsensusClusterPlus: a class discovery tool with confidence assessments and item tracking. Bioinformatics26, 1572-1573, doi:10.1093/bioinformatics/btq170 (2010).

Vogt, B. A., Nimchinsky, E. A., Vogt, L. J. \& Hof, P. R. Human cingulate cortex: surface features, flat maps, and cytoarchitecture. J Comp Neuro/359, 490-506, doi:10.1002/cne.903590310 (1995).

Hubbard, E. M. \& Ramachandran, V. S. Neurocognitive mechanisms of synesthesia. Neuron48, 509-520, doi:10.1016/j.neuron.2005.10.012 (2005).

Dehaene, S. \& Cohen, L. The unique role of the visual word form area in reading. Trends Cogn Sci15, 254-262, doi:10.1016/j.tics.2011.04.003 (2011).

Meng, M., Cherian, T., Singal, G. \& Sinha, P. Lateralization of face processing in the human brain. Proc Biol Sci279, 2052-2061, doi:10.1098/rspb.2011.1784 (2012).

Aminoff, E. M., Kveraga, K. \& Bar, M. The role of the parahippocampal cortex in cognition. Trends Cogn Sci17, 379-390, doi:10.1016/j.tics.2013.06.009 (2013).

Valk, J. A Possible Role of BA8 in Pre-surgical fMRI. Homage to an Exceptional Neuroscientist. Neuroradiol J24, 395-400, doi:10.1177/197140091102400308 (2011).

Gered, S. Understanding Consciousness: Its Function and Brain Processes. SAGE Publications, 135-156 (2000).

Li, P. et al. Structural and functional brain network of human retrosplenial cortex. Neurosci Lett674, 24-29, doi:10.1016/j.neulet.2018.03.016 (2018). 
Ardila, A., Bernal, B. \& Rosselli, M. How Extended Is Wernicke's Area? Meta-Analytic Connectivity Study of BA2O and Integrative Proposal. Neurosci J2016, 4962562, doi:10.1155/2016/4962562 (2016).

21 Berthier, M. L., Pulvermuller, F., Davila, G., Casares, N. G. \& Gutierrez, A. Drug therapy of post-stroke aphasia: a review of current evidence. Neuropsychol Rev21, 302-317, doi:10.1007/s11065-011-9177-7 (2011).

22 Movsessian, P. Neuropharmacology of theophylline induced stuttering: the role of dopamine, adenosine and GABA. Med Hypotheses64, 290-297, doi:10.1016/j.mehy.2004.07.026 (2005).

23 Bergmann, S., Ihmels, J. \& Barkai, N. Iterative signature algorithm for the analysis of large-scale gene expression data. Phys Rev E Stat Nonlin Soft Matter Phys67, 031902, doi:10.1103/PhysRevE.67.031902 (2003).

24 Leech, R. \& Sharp, D. J. The role of the posterior cingulate cortex in cognition and disease. Brain137, 12-32, doi:10.1093/brain/awt162 (2014).

Joyce, M. K. P. \& Barbas, H. Cortical Connections Position Primate Area 25 as a Keystone for Interoception, Emotion, and Memory. J Neurosci38, 1677-1698, doi:10.1523/JNEUROSCI.2363-17.2017 (2018).

26 Basu, J. et al. Gating of hippocampal activity, plasticity, and memory by entorhinal cortex long-range inhibition. Science351, aaa5694, doi:10.1126/science.aaa5694 (2016).

27 Min, S. W. et al. Critical role of acetylation in tau-mediated neurodegeneration and cognitive deficits. Nat Med21, 1154-1162, doi:10.1038/nm.3951 (2015).

28 Jacobs, H. I. L. et al. Structural tract alterations predict downstream tau accumulation in amyloid-positive older individuals. Nat Neurosci21, 424-431, doi:10.1038/s41593-018-0070-z (2018).

29 Lim, Y. et al. alpha-Syn suppression reverses synaptic and memory defects in a mouse model of dementia with Lewy bodies. I Neurosci31, 10076-10087, doi:10.1523/JNEUROSCI.0618-11.2011 (2011).

30 Rioult-Pedotti, M. S., Pekanovic, A., Atiemo, C. O., Marshall, J. \& Luft, A. R. Dopamine Promotes Motor Cortex Plasticity and Motor Skill Learning via PLC Activation. PLoS One10, e0124986, doi:10.1371/journal.pone.0124986 (2015).

31 Bakhshishayan, S. et al. Protein kinase A regulates the long-term potentiation of intrinsic excitability in neonatal trigeminal motoneurons. Brain Res1541, 1-8, doi:10.1016/j.brainres.2013.10.010 (2013).

Isensee, J. et al. Pain modulators regulate the dynamics of PKA-RII phosphorylation in subgroups of sensory neurons. J Cell Sci127, 216-229, doi:10.1242/jcs.136580 (2014).

33 Herrmann, S. et al. Protein kinase A regulates inflammatory pain sensitization by modulating HCN2 channel activity in nociceptive sensory neurons. Pain158, 2012-2024, doi:10.1097/j.pain.0000000000001005 (2017).

34 Chagniel, L., Bergeron, Y., Bureau, G., Massicotte, G. \& Cyr, M. Regulation of tyrosine phosphatase STEP61 by protein kinase A during motor skill learning in mice. PLOS One9, e86988, doi:10.1371/journal.pone.0086988 (2014).

35 Canafoglia, L. et al. Sensorimotor cortex excitability in Unverricht-Lundborg disease and Lafora body disease. Neurology63, 2309-2315 (2004).

Lake, N. J. et al. Biallelic Mutations in MRPS34 Lead to Instability of the Small Mitoribosomal Subunit and Leigh Syndrome. Am J Hum Genet101, 239-254, doi:10.1016/j.ajhg.2017.07.005 
(2017).

7 Chahrour, M. et al. MeCP2, a key contributor to neurological disease, activates and represses transcription. Science320, 1224-1229, doi:10.1126/science.1153252 (2008).

38 Krishnan, K. et al. MeCP2 regulates the timing of critical period plasticity that shapes functional connectivity in primary visual cortex. Proc Natl Acad Sci U S A112, E4782-4791, doi:10.1073/pnas.1506499112 (2015).

39 Durand, S. et al. NMDA receptor regulation prevents regression of visual cortical function in the absence of Mecp2. Neuron76, 1078-1090, doi:10.1016/j.neuron.2012.12.004 (2012).

40 Uhlen, M. et al. Proteomics. Tissue-based map of the human proteome. Science347, 1260419, doi:10.1126/science.1260419 (2015).

41 Turner, M. R. et al. Distinct cerebral lesions in sporadic and 'D90A' SOD1 ALS: studies with [11C]flumazenil PET. Brain128, 1323-1329, doi:10.1093/brain/awh509 (2005).

42 White, J. K. et al. Genome-wide generation and systematic phenotyping of knockout mice reveals new roles for many genes. Cell154, 452-464, doi:10.1016/j.cell.2013.06.022 (2013).

43 Wang, Y., Matsuzaka, Y., Mushiake, H. \& Shima, K. Spatial distribution of cingulate cortical cells projecting to the primary motor cortex in the rat. Neurosci Res60, 406-411, doi:10.1016/j.neures.2007.12.012 (2008).

44 Caruana, F. et al. Motor and emotional behaviours elicited by electrical stimulation of the human cingulate cortex. Brain141, 3035-3051, doi:10.1093/brain/awy219 (2018).

45 Hof, P. R., Nimchinsky, E. A. \& Morrison, J. H. Neurochemical phenotype of corticocortical connections in the macaque monkey: quantitative analysis of a subset of neurofilament protein-immunoreactive projection neurons in frontal, parietal, temporal, and cingulate cortices. J Comp Neuro/362, 109-133, doi:10.1002/cne.903620107 (1995).

46 Jacomy, H., Zhu, Q., Couillard-Despres, S., Beaulieu, J. M. \& Julien, J. P. Disruption of type IV intermediate filament network in mice lacking the neurofilament medium and heavy subunits. J Neurochem73, 972-984 (1999).

47 Rao, M. V. et al. Neurofilament-dependent radial growth of motor axons and axonal organization of neurofilaments does not require the neurofilament heavy subunit (NF-H) or its phosphorylation. J Cell Bio/143, 171-181 (1998).

48 McGraw, T. S., Mickle, J. P., Shaw, G. \& Streit, W. J. Axonally transported peripheral signals regulate alpha-internexin expression in regenerating motoneurons. J Neurosci22, 4955-4963 (2002).

49 Hyung, S. et al. Optogenetic neuronal stimulation promotes axon outgrowth and myelination of motor neurons in a three-dimensional motor neuron-Schwann cell coculture model on a microfluidic biochip. Biotechnol Bioeng116, 2425-2438, doi:10.1002/bit.27083 (2019). Shvil, N. et al. MIF inhibits the formation and toxicity of misfolded SOD1 amyloid aggregates: implications for familial ALS. Cell Death Dis9, 107, doi:10.1038/s41419-017-0130-4 (2018).

51 Mattson, M. P. Calcium and neurodegeneration. Aging Cell6, 337-350, doi:10.1111/j.1474-9726.2007.00275.x (2007).

52 Nijssen, J., Comley, L. H. \& Hedlund, E. Motor neuron vulnerability and resistance in amyotrophic lateral sclerosis. Acta Neuropatho/133, 863-885, doi:10.1007/s00401-017-1708-8 (2017).

53 Kessler, R. C. et al. The global burden of mental disorders: an update from the WHO World Mental Health (WMH) surveys. Epidemiol Psichiatr Soc18, 23-33 (2009). 


\begin{tabular}{|c|c|c|}
\hline $\begin{array}{l}885 \\
886\end{array}$ & 54 & $\begin{array}{l}\text { Alonso, J. et al. Treatment gap for anxiety disorders is global: Results of the World Mental } \\
\text { Health Surveys in } 21 \text { countries. Depress Anxiety35, 195-208, doi:10.1002/da.22711 (2018). }\end{array}$ \\
\hline 887 & 55 & Pallanti, S. et al. Motor cortex excitability correlates with novelty seeking in social anxiety: a \\
\hline 888 & & transcranial magnetic stimulation investigation. J Neuro/257, \\
\hline 889 & & doi:10.1007/s00415-010-5533-4 (2010). \\
\hline 890 & 56 & Wassermann, E. M., Greenberg, B. D., Nguyen, M. B. \& Murphy, D. L. Motor cortex excitability \\
\hline 891 & & correlates with an anxiety-related personality trait. Biol Psychiatry50, 377-382, \\
\hline 892 & & doi:10.1016/s0006-3223(01)01210-0 (2001). \\
\hline 893 & 57 & Shin, J. et al. Phospholipase $C$ beta 4 in the medial septum controls cholinergic theta \\
\hline 894 & & oscillations and anxiety behaviors. $J$ Neurosci29, 15375-15385, \\
\hline 895 & & doi:10.1523/JNEUROSCI.3126-09.2009 (2009). \\
\hline 896 & 58 & Zhang, J. et al. Neuronal nitric oxide synthase alteration accounts for the role of $5-\mathrm{HT} 1 \mathrm{~A}$ \\
\hline 897 & & receptor in modulating anxiety-related behaviors. $J$ Neurosci30, 2433-2441, \\
\hline 898 & & doi:10.1523/JNEUROSCI.5880-09.2010 (2010). \\
\hline 899 & 59 & Mo, J., Wang, B., Zhu, X., Wu, X. \& Liu, Y. PRRT2 deficiency induces paroxysmal kinesigenic \\
\hline 900 & & dyskinesia by influencing synaptic function in the primary motor cortex of rats. Neurobiol \\
\hline 901 & & Dis121, 274-285, doi:10.1016/j.nbd.2018.10.011 (2019). \\
\hline 902 & 60 & Ryan, T. J. et al. Evolution of GluN2A/B cytoplasmic domains diversified vertebrate synaptic \\
\hline 903 & & plasticity and behavior. Nat Neurosci16, 25-32, doi:10.1038/nn.3277 (2013). \\
\hline 904 & 61 & Vekovischeva, O. Y. et al. Reduced aggression in AMPA-type glutamate receptor GluR-A \\
\hline 905 & & subunit-deficient mice. Genes Brain Behav3, 253-265, doi:10.1111/j.1601-1848.2004.00075.x \\
\hline 906 & & (2004). \\
\hline 907 & 62 & Lv, Y. et al. Whole-exome sequencing identifies a donor splice-site variant in SMPX that causes \\
\hline 908 & & rare X-linked congenital deafness. Mol Genet Genomic Med7, e967, doi:10.1002/mgg3.967 \\
\hline 909 & & (2019). \\
\hline 910 & 63 & Likova, L. T. Addressing long-standing controversies in conceptual knowledge representation \\
\hline 911 & & in the temporal pole: A cross-modal paradigm. IS\&T Int Symp Electron Imaging2017, 268-272, \\
\hline 912 & & doi:10.2352/ISSN.2470-1173.2017.14.HVEI-155 (2017). \\
\hline 913 & 64 & Ardila, A., Bernal, B. \& Rosselli, M. How Localized are Language Brain Areas? A Review of \\
\hline 914 & & Brodmann Areas Involvement in Oral Language. Arch Clin Neuropsycho/31, 112-122, \\
\hline 915 & & doi:10.1093/arclin/acv081 (2016). \\
\hline 916 & 65 & Giraud, A. L. et al. Contributions of sensory input, auditory search and verbal comprehension \\
\hline 917 & & to cortical activity during speech processing. Cereb Cortex14, 247-255, \\
\hline 918 & & doi:10.1093/cercor/bhg124 (2004). \\
\hline 919 & 66 & Dienel, G. A. Brain Glucose Metabolism: Integration of Energetics with Function. Physiol \\
\hline 920 & & Rev99, 949-1045, doi:10.1152/physrev.00062.2017 (2019). \\
\hline & 67 & Manza, P., Zhang, S., Li, C. S. \& Leung, H. C. Resting-state functional connectivity of the \\
\hline & & striatum in early-stage Parkinson's disease: Cognitive decline and motor symptomatology. \\
\hline & & Hum Brain Mapp37, 648-662, doi:10.1002/hbm.23056 (2016). \\
\hline & 68 & Wei, L., Hu, X., Yuan, Y., Liu, W. \& Chen, H. Abnormal ventral tegmental area-anterior \\
\hline & & cingulate cortex connectivity in Parkinson's disease with depression. Behav Brain Res347, \\
\hline 926 & & 132-139, doi:10.1016/j.bbr.2018.03.011 (2018). \\
\hline & 69 & Hao, X. et al. Urinary protein biomarkers for pediatric medulloblastoma. J Proteomics $\mathbf{2 2 5}$, \\
\hline & & oi:10.1016/j.jprot.2020.103832 (2020). \\
\hline
\end{tabular}


bioRxiv preprint doi: https://doi.org/10.1101/2021.03.07.434254; this version posted March 7, 2021. The copyright holder for this preprint (which was not certified by peer review) is the author/funder. All rights reserved. No reuse allowed without permission.

92970 Anders, S. \& Huber, W. Differential expression analysis for sequence count data. Genome 930 Biol11, R106, doi:10.1186/gb-2010-11-10-r106 (2010).

931

932 
933 Table 1: Location, function and proteomic clustering of 29 human brain Brodmann

934 areas.

\begin{tabular}{|c|c|c|c|c|}
\hline Cluster & $\begin{array}{l}\text { Brodmann } \\
\text { area }\end{array}$ & Lobe & Location & Function \\
\hline \multirow{7}{*}{ Motor and sensory } & $3 / 1 / 2$ & Parietal Lobe & Postcentralgyrus & Somatosensory system ${ }^{1}$ \\
\hline & 4 & Frontal Lobe & Precentralgyrus & Primary motor cortex ${ }^{2}$ \\
\hline & 6 & Frontal Lobe & Frontal Lobe & $\begin{array}{l}\text { Premotor cortex and supplementary motor } \\
\qquad \text { cortex }^{2}\end{array}$ \\
\hline & 20 & Temporal Lobe & Inferior temporal gyrus & Visual processing and recognition ${ }^{3}$ \\
\hline & 38 & Temporal Lobe & Temporal pole & Visceral emotional responses ${ }^{4}$ \\
\hline & 17 & Occipital Lobe & Occipital Lobe & Visual processing $^{5}$ \\
\hline & 18 & Occipital Lobe & Occipital Lobe & Visual processing $^{5}$ \\
\hline \multirow{5}{*}{ Visual } & 19 & Occipital Lobe & Occipital Lobe & Visual processing $^{5}$ \\
\hline & 37 & Temporal Lobe & Fusiform gyrus & Visual recognition ${ }^{6-8}$ \\
\hline & 34 & Temporal lobe & Parahippocampalgyrus & $\begin{array}{c}\text { Neuron information processing; visual } \\
\text { processing } \\
\text { pros }^{9}\end{array}$ \\
\hline & 8 & Frontal Lobe & Prefrontal cortex & Eye movements; writing language ${ }^{10}$ \\
\hline & 7 & Parietal Lobe & Parietal Lobe & $\begin{array}{l}\text { Visuo-motor coordination; speech and } \\
\text { language }\end{array}$ \\
\hline \multirow[t]{3}{*}{ Auditory and Broca's area } & $26 / 29 / 30$ & Cingulate cortex & Retrosplenial cortex & Cognition;memory $^{12}$;uditory $^{13}$ \\
\hline & $41 / 42$ & Temporal Lobe & $\begin{array}{l}\text { Anterior transverse } \\
\text { temporal gyrus }\end{array}$ & Auditory cortex ${ }^{14}$ \\
\hline & $44 / 45$ & Frontal Lobe & Inferior frontal gyrus & Broca'sarea, language production ${ }^{15}$ \\
\hline Heterogeneous functions & 9 & Frontal Lobe & $\begin{array}{l}\text { Dorsolateral prefrontal } \\
\text { cortex }\end{array}$ & Executive functions, cognition, behaviour ${ }^{16}$ \\
\hline
\end{tabular}


10 Frontal Lobe Anterior prefrontal cortex Executive functions, cognition, memory ${ }^{17-19}$

$39 \quad$ Parietal Lobe Angular gyrus

Frontal Lobe

21 Temporal Lobe

Wernicke's area

Cingulate cortex

Cingulate cortex

Ventral Posterior cingulate

Cingulate cortex
Temporal Lobe cortex

Ventral anterior cingulate cortex

Subgenual cingulate

Middle temporal gyrus

Wernicke's area, comprehension of written and spoken language ${ }^{28}$

Wernicke's area, comprehension of written and spoken language ${ }^{28}$

Emotion and memory; intrinsic control

$$
\text { networks }^{29}
$$

Emotional and cognitive processing $\mathrm{g}^{30,31}$

Emotional and cognitive processing ${ }^{30,31}$

Emotion and memory; intrinsic control networks $^{29}$

Emotional and cognitive processing $g^{30,31}$

Emotion and memory ${ }^{32}$

Ventral entorhinal cortex 
9371 Viaene, A. N., Petrof, I. \& Sherman, S. M. Synaptic properties of thalamic input to layers $2 / 3$ 938 and 4 of primary somatosensory and auditory cortices. J Neurophysiol105, 279-292, doi:10.1152/jn.00747.2010 (2011).

9402 Zilles, K. \& Amunts, K. Centenary of Brodmann's map--conception and fate. Nat Rev

$941 \quad$ Neurosci11, 139-145, doi:10.1038/nrn2776 (2010).

9423 Haxby, J. V., Hoffman, E. A. \& Gobbini, M. I. The distributed human neural system for face 943 perception. Trends Cogn Sci4, 223-233 (2000).

9444 Ding, S. L., Van Hoesen, G. W., Cassell, M. D. \& Poremba, A. Parcellation of human temporal 945 polar cortex: a combined analysis of multiple cytoarchitectonic, chemoarchitectonic, and pathological markers. J Comp Neurol514, 595-623, doi:10.1002/cne.22053 (2009). www.lifesci.sussex.ac.uk/home/George_Mather/Linked\%20Pages/Physiol/Cortex.html. 509-520, doi:10.1016/j.neuron.2005.10.012 (2005).

Dehaene, S. \& Cohen, L. The unique role of the visual word form area in reading. Trends Cogn Sci15, 254-262, doi:10.1016/j.tics.2011.04.003 (2011).

Meng, M., Cherian, T., Singal, G. \& Sinha, P. Lateralization of face processing in the human brain. Proc Biol Sci279, 2052-2061, doi:10.1098/rspb.2011.1784 (2012).

Aminoff, E. M., Kveraga, K. \& Bar, M. The role of the parahippocampal cortex in cognition. Trends Cogn Sci17, 379-390, doi:10.1016/j.tics.2013.06.009 (2013).

Valk, J. A Possible Role of BA8 in Pre-surgical fMRI. Homage to an Exceptional Neuroscientist. Neuroradiol J24, 395-400, doi:10.1177/197140091102400308 (2011).

Gered, S. Understanding Consciousness: Its Function and Brain Processes. SAGE Publications, 135-156 (2000).

Vann, S. D., Aggleton, J. P. \& Maguire, E. A. What does the retrosplenial cortex do? Nat Rev Neurosci10, 792-802, doi:10.1038/nrn2733 (2009).

Li, P. et al. Structural and functional brain network of human retrosplenial cortex. Neurosci Lett674, 24-29, doi:10.1016/j.neulet.2018.03.016 (2018).

Pickles, J. O. An Introduction to the Physiology of Hearing 4th ed. edn, 238 (Emerald Group Publishing Limited).

Cantalupo, C. \& Hopkins, W. D. Asymmetric Broca's area in great apes. Nature414, 505, doi:10.1038/35107134 (2001).

6 Hoshi, E. Functional specialization within the dorsolateral prefrontal cortex: a review of anatomical and physiological studies of non-human primates. Neurosci Res54, 73-84, doi:10.1016/j.neures.2005.10.013 (2006).

Ramnani, N. \& Owen, A. M. Anterior prefrontal cortex: insights into function from anatomy and neuroimaging. Nat Rev Neurosci5, 184-194, doi:10.1038/nrn1343 (2004).

Semendeferi, K., Armstrong, E., Schleicher, A., Zilles, K. \& Van Hoesen, G. W. Prefrontal cortex in humans and apes: a comparative study of area 10. Am J Phys Anthropol114, 224-241, doi:10.1002/1096-8644(200103)114:3<224::AID-AJPA1022>3.0.CO;2-I (2001).

Gilbert, S. J. et al. Functional specialization within rostral prefrontal cortex (area 10): a meta-analysis. J Cogn Neurosci18, 932-948, doi:10.1162/jocn.2006.18.6.932 (2006).

0 Seghier, M. L. The angular gyrus: multiple functions and multiple subdivisions. Neuroscientist19, 43-61, doi:10.1177/1073858412440596 (2013). 
1001

1002

1003

1004

1005

1006

1007

1008

1009

1010

1011

1012

1013

1014

1015
21 Brownsett, S. L. \& Wise, R. J. The contribution of the parietal lobes to speaking and writing. Cereb Cortex20, 517-523, doi:10.1093/cercor/bhp120 (2010).

22 Grabner, R. H. et al. Individual differences in mathematical competence predict parietal brain activation during mental calculation. Neuroimage38, 346-356, doi:10.1016/j.neuroimage.2007.07.041 (2007).

23 Chen, Q., Weidner, R., Vossel, S., Weiss, P. H. \& Fink, G. R. Neural mechanisms of attentional reorienting in three-dimensional space. $J$ Neurosci32, 13352-13362, doi:10.1523/JNEUROSCI.1772-12.2012 (2012).

24 Dehaene, S., Spelke, E., Pinel, P., Stanescu, R. \& Tsivkin, S. Sources of mathematical thinking: behavioral and brain-imaging evidence. Science 284, 970-974 (1999).

Hirnstein, M., Bayer, U., Ellison, A. \& Hausmann, M. TMS over the left angular gyrus impairs the ability to discriminate left from right. Neuropsychologia49, 29-33, doi:10.1016/j.neuropsychologia.2010.10.028 (2011).

Stoeckel, C., Gough, P. M., Watkins, K. E. \& Devlin, J. T. Supramarginal gyrus involvement in visual word recognition. Cortex45, 1091-1096, doi:10.1016/j.cortex.2008.12.004 (2009).

7 Gazzaniga, M. S., Ivry, R.B. and Mangun, G.R. Cognitive Neuroscience, the Biology of the Mind. third edition edn, 395-401 (W.W. Norton,, 2009).

28 Ardila, A., Bernal, B. \& Rosselli, M. How Extended Is Wernicke's Area? Meta-Analytic Connectivity Study of BA20 and Integrative Proposal. Neurosci J2016, 4962562, doi:10.1155/2016/4962562 (2016).

29 Leech, R. \& Sharp, D. J. The role of the posterior cingulate cortex in cognition and disease. Brain137, 12-32, doi:10.1093/brain/awt162 (2014).

30 Bush, G., Luu, P. \& Posner, M. I. Cognitive and emotional influences in anterior cingulate cortex. Trends Cogn Sci4, 215-222 (2000).

31 Allman, J. M., Hakeem, A., Erwin, J. M., Nimchinsky, E. \& Hof, P. The anterior cingulate cortex. The evolution of an interface between emotion and cognition. Ann N Y Acad Sci935, 107-117 (2001).

32 Joyce, M. K. P. \& Barbas, H. Cortical Connections Position Primate Area 25 as a Keystone for Interoception, Emotion, and Memory. J Neurosci38, 1677-1698, doi:10.1523/JNEUROSCI.2363-17.2017 (2018).

33 Basu, J. et al. Gating of hippocampal activity, plasticity, and memory by entorhinal cortex long-range inhibition. Science351, aaa5694, doi:10.1126/science.aaa5694 (2016). 
1017

\section{Figure legends}

Figure 1:A broad and comprehensive atlas of brain proteome covering 29 BAs. A: Graphical illustration of the workflow for brain proteome database in Brodmann Area (BA46) and brain proteomic map in the 29 major BAs. First, an extensive proteomic library was generated of BA46by 2D-LC-MS/MS and label-free quantification in BA46. Then, 29 BAs were quantitatively analyzed by 2D-LC-MS/MS and iTRAQ quantification. Further, a website, Human Brain Proteome Atlas, HBPA(www.brain-omics.com) was built. B: Venn diagram of protein number of BA46 in present and previous studies. C: Accumulation of protein mass from the highest abundance to the lowest abundance protein in BA46. The high abundance proteins, (grey, top for $75 \%$ of total protein abundance), mid abundance proteins, (orange, top $75 \%-95 \%$ of total protein abundance), and low abundance proteins (blue, bottom $5 \%$ total protein amount) shown by distinct colors. D: GO enrichment analysis of the brain proteome, and the high, middle, low abundance brain proteins, and compared with the whole proteome. The percentages of the proteins in each category were in bar plot. *: $\mathrm{p}<0.05$.

Figure 2: Brain proteomic atlas reflects the functional parcellation. A: The 29 brain areas were divided into 6 clusters, including motor and sensory cluster, vision cluster, auditory and Broca's area cluster, Wernicke's area cluster, cingulate cortex cluster, and heterogeneous function cluster. The pearson correlation heatmap of 29 BAs in the left. Red: positive correlation; Blue: negative correlation. The locations of 29 BAs were shown in the right. B: Brief annotations of 29 BAs in the 6 clusters. The lobe location of each BA was annotated in the inner circle, and the function of each BA was annotated in the outer circle. Bold: Canonical function; Italic: uncanonical function.

Figure 3:Cluster signature proteins linked to specialized function of the cluster. A: Heat map of the cluster signature proteins. The normalized protein expression value z-score represents the protein abundance. Red: high abundance. Blue: low abundance. 
B,C: Pathway (B) and disease (C) enrichment analysis of cluster signature proteins in six clusters. The -Log (p-value) by two-sided hypergeometric test were in heatmaps.Red:significantly enriched. D: Distribution of the high-, mid-, lowabundance proteins in the cluster signature proteins. The number of high-, mid-, lowabundance proteins in each cluster signature proteins were annotated in the bar plot. E: Heatmap of cingulate cortex cluster signature proteins involved in glycolysis/gluconeogenesis, oxidative phosphorylation, and biosynthesis of amino acids. The normalized protein expression value z-score represents the protein abundance. Red: high abundance. Blue: low abundance. F: The relative expression value of two motor and sensory cluster signature proteins (MRPS34 and EMP2A) and two cingulate cortex cluster specific proteins (SYUA and MAPT) in all the 29 BAs. The motor and sensory cluster and the cingulate cortex cluster were highlighted by yellow in the left and right panels, respectively.

Figure 4: BA signature proteins linked to BAs' function. A: Heat map of the BA signature proteins in 29 BAs. The normalized protein expression value z-score represents the protein abundance. Red: high abundance. Blue: low abundance. B: The relative expression value of three BA signature proteins, TAGLN, RAD23A, and FAM107B in all the 29 BAs. The BA41/42, BA40, BA17 and BA18 were highlighted by yellow in the upper, middle, and lower panels, respectively.

Figure 5: Molecular annotation of the un-canonical functions of BAs. A: Scatter plot of protein $\log 2$ fold expression value in BA 33 compared with the value in BA4. Proteins expressed at high level in both BA4 and BA33 highlighted in orange. B: The co-highly-expressed proteins in BA4 and BA33 were associated with motor neuron structure and functions. C: Clustering analysis of anxiety-related proteins. The proteins highly expressed in motor and sensory cluster highlighted in red. D: Protein network of anxiety-related proteins. Orange: The proteins expressed at high level in motor and sensory cluster. Green:The proteins expressed at low level in motor and sensory cluster. 
1073 Figure 6: Comparison of brain proteome and transcriptome.A: Venn diagram of 1074 protein and mRNA number in BA46. B: Histogram of the distribution of the gene 1075 expression in mRNA and protein level. Orange: Gene identified in protein level. Grey: 1076 Genes only identified only in mRNA level. The MS/MS coding genes covered $61 \%$ 1077 genes expressed at $>1$ read per kilobase per million mapped (RPKM)and78.4\% of 1078 genes expressed at >10 RPKM. C: Principal component analysis of mRNA shows that 1079 the BA4 and cluster cingulate cortex were partially separated with the other BAs. D: 1080 Heat map of the BA signature genes in 27 BAs. The normalized protein expression 1081 value $\mathrm{z}$-score represents the protein abundance. Red: high abundance. Blue: low 1082 abundance. E: The Distribution of the relative dominance of no change (grey), agree 1083 (green), partially agree (purple), protein only (Blue), mRNA only (orange), and 1084 disagree (red)genes.Genes are defined based on the consistency or inconsistency 1085 between the mRNA and protein measurements. Agree genes are those with same 1086 direction of change in both RNA and protein level, and with the same magnitude of 1087 fold change (the variability in protein level between BAs is < 2 fold of that in mRNA 1088 level). Partially agree genes are genes with same direction of change, but with 1089 different magnitude of fold change (larger than 2 fold) in RNA and protein level. No 1090 change genes, both protein and RNA were not changed (within 2 fold) between the BAs. Protein only genes vary between different BAs by protein (larger than 2 fold), 1092 but not by mRNA (within 2 fold). mRNA only genes vary by mRNA (larger than 2 fold), but not by protein (within 2 fold). Disagree genes performed opposite direction 1094 of change between protein and mRNA. F:Scatter plots of $\log 2$ formed mRNA and 1095 protein abundance fold change between BA4 and BA46. grey (no change), Green 1096 (agree), purple (partially agree), Blue (protein only), orange (mRNA only), and Red 1097 (disagree) genes were defined in E. The inset pie charts illustrated the percentage of 1098 the green, purple,blue, orange, and red genes. G: Scatter plots of mRNA and protein 1099 fold change for BA4/ BA46 comparison, showing the position of gene products 1100 contained in ontological terms of interest. The inset small table showed the percentage 1101 of the genes of responding ontological terms in the mRNA only, protein only, agree, 
1102 partially agree and disagree categories. The colored lines indicated the threshold of 1103 mRNA gene (orange), protein only gene (blue), and the agree gene (green). 


\section{Supplementary material}

1106

1107

1108

1109

1110

1111

1112

1113

1114

1115

1116

1117

1118

1119

1120

1121

1122

1123

1124

1125

1126

1127

1128

1129

1130

1131

1132

1133

1134

1135

1136

1137

1138

\section{Supplementary Figure 1: The HE staining data of two brains. A: Brain 1; B:} Brain 2.

Supplementary Figure 2: Reliability of iTRAQ analysis pipeline. A: Technical CV distributions of label-free and iTRAQ quantification. B: The venn diagram of the proteins quantified in 4 batches. C: Log ratio distribution of iTRAQ quantification results. D: The ratio distributions of each run in 4 batches.

Supplementary Figure 3: Protein abundance distribution of BA46 from the highest abundance to the lowest abundance protein.

\section{Supplementary Figure 4:Data visualization in Human Brain Proteome Atlas} website. A: Protein quantification distribution of MAPT in 29 Brodmann areas. B: Protein expression data in Brodmann area 25( Subgenual area). C: Comparison of the differential proteins between BA3/1/2, and BA4.

Supplementary Figure 5: The protein clustering analysis. A: The clustering is derived by consensus clustering based on 1,000 resampled data sets, exploring the range of from $\mathrm{k}=2$ to $\mathrm{k}=8$. $\mathrm{B}$ : Based on both consensus cumulative distribution function $(\mathrm{CDF})$ area curve and the delta plot assessing change in CDF area, the CDF of consensus increased at greater $\mathrm{k}$ values. At $\mathrm{k}=6$, a relative increase in CDF reached a very small value. . C: Silhouette plots were performed to evaluate the Stability of the clustering. The silhouette plot at $\mathrm{k}=6$ showed a mean silhouette score of 0.72 , and all scores were positive.

Supplementary Figure 6: Analysis of the differential proteins between Wernicke's area cluster and auditory and Broca's area cluster. A: IPA-based Canonical pathway analysis of differential proteins. The $-\log$ (p-value) of the Canonical pathway term were shown. B: Heatmap of differential proteins involved in language eloquent regions related neurotransmitter pathways. The normalized protein expression value z-score represents the protein abundance. Red: high abundance. Blue: low abundance.

Supplementary Figure 7: Functional analysis of cluster signature proteins, related to Figure 3. A: The IPA-based biofunction (A) and KEGG database-based pathway (B) enrichment analysis of cluster signature proteins. The -Log (p-value) by two-sided hypergeometric test in heatmap. Red: significantly enriched. C-E: Heat map of the high-abundance proteins (C), mid-abundance proteins (D) and low abundance proteins(E) in the 29 BAs. The normalized protein expression value z-score represents 
1139 the protein abundance. Red: high abundance. Blue: low abundance. F: Protein

1140

1141

1142

1143

1144

1145

1146

1147

1148

1149

1150

1151

1152

1153

1154

1155

1156

1157

1158

1159

1160 expression analysis of cingulate cortex cluster in Parkinson's signaling. G: Protein expression analysis of cingulate cortex cluster in Alzheimer's disease signaling. (Red: highly-expressed in cingulate cortex cluster; Green: lowly-expressed in cingulate cortex cluster)

\section{Supplementary Figure 8: The Western Blot and IHC-Fr results of cluster} signature proteins. A: Western Blot results of two motor and sensory cluster signature proteins (MRPS34 and EPM2A) and two cingulate cortex cluster signature proteins (Tau and a-syn). B: Comparison between immunoblotting results and iTRAQ results of above proteins. C: IHC-Fr validation results of two cingulate cortex cluster signature proteins (MAPT and SYUA).Scale bars represent as $50 \mu \mathrm{m}$.

\section{Supplementary Figure 9: The Western Blot and IHC-Frresults of Brodmann} area signature proteins. A:Western Blot results of three Brodmann area signature proteins (Transgelin, FAM107B, and RAD23A). B: Comparison between the immunoblotting results and iTRAQ results of above proteins.

Supplementary Figure 10: Quality control of RNA-seq. A: Number of gene detected in RNA-seq analysis of the 21 BAs. B: Box plot of gene expression level of 21 BAs.

Supplementary Figure 11: Scatter plots of mRNA and protein fold change of all regions compared with $\mathrm{BA}$ 46. These scatter plots are identically defined as those in Figure 6E. 
1161 Supplementary Table 1: Brain proteome database in BA46.A:Qualitative data of

1162 BA46 technical repeat 1, 2 and 3. The proteins identified in Kim's study and Becky's

1163 study were annotated. B: Protein identification summary of BA 46. C: Quantitative

1164 data of BA 46. D: The GO analysis results of brain proteome and high-, mid-, and

1165 low-abundance proteins compared with the whole human proteome.

1166

1167

1168

1169

1170

1171

1172

1173

1174

1175

1176

1177

1178

1179

1180

1181

1182

1183

1184

1185

1186

1187

1188

Supplementary Table 2: iTRAQ quantification data for 29 BAs. A: Batch 1; B:

Batch 2; C: Batch 3; D: Batch 4. E: Protein identification summary of the human brain proteome of 29 BAs. F: Normalized protein quantification data of the 4308 proteins in all of 29 BAs. The 1241 proteins with inter-area variability (the difference between the maximum and minimum ratios $\geq 2$ ) were annotated.

\section{Supplementary Table 3: Differentially proteins between the Wernicke's area} cluster and Auditory and Broca's area cluster.

Supplementary Table 4: Cluster/BA signature proteins. A:List of cluster signature proteins. B: Biofunction analysis of cluster signature proteins. C: Disease analysis of cluster signature proteins. D: Canonical pathway analysis of cluster signature proteins. E: KEGG pathway analysis of cluster signature proteins. F: Cluster cingulate cortex signature proteins were involved in cognition, emotion, and memory. G: BA signature proteins.

Supplementary Table 5: Validation of the Cluster/BA signature proteins. A: PRM results of Cluster signature proteins (peptide level). B: PRM results of Cluster signature proteins (protein level). C: PRM results of BA signature proteins (peptide level). D: PRM results of BA signature proteins (protein level). E: Comparison of Vision cluster/vision cortex signature proteins results between this study and Becky's study.

Supplementary Table 6: Molecular annotation of the un-canonical functions of BAs. A: Proteins highly expressed in both Primary motor cortex (BA4) and Anterior cingulate cortex (BA33). B:Thirty proteins with inter-area variability were involved in anxiety or anxiety-like behaviors. 


\section{Supplementary Table 7. Comparison of brain proteome and}

1190 transcriptome.A:RNA-seq results for the 21 BAs. B: BA signature genes. C:Thirteen

1191 signature genes involved in memoryin BA28. D. The common genes in transcriptomic 1192 and proteomic results. E: The differential transcriptomic and proteomic results for 1193 BA4 and BA46 comparison. F. The disease and biofunction analysis of differential 1194 genes of BA4 and BA46. The categories mentioned in the manuscript were 1195 highlighted in yellow. 


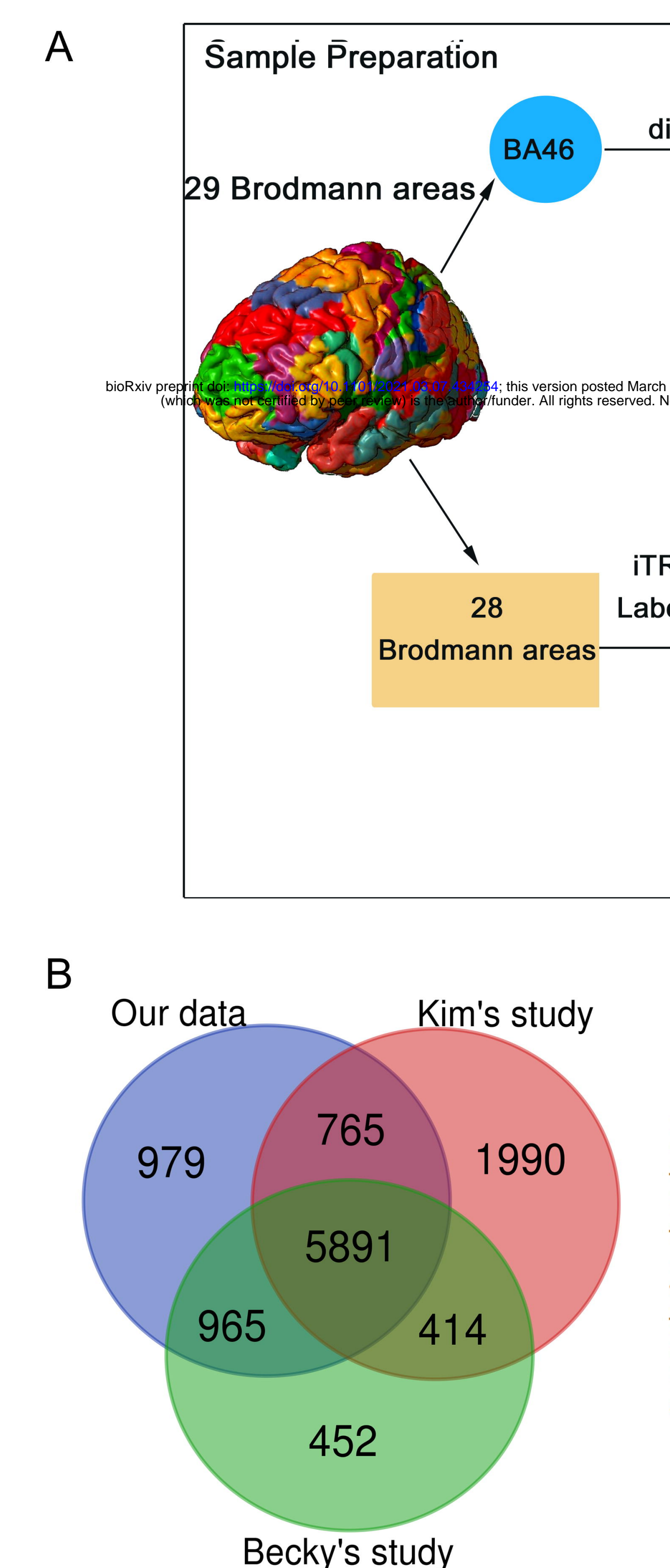

C

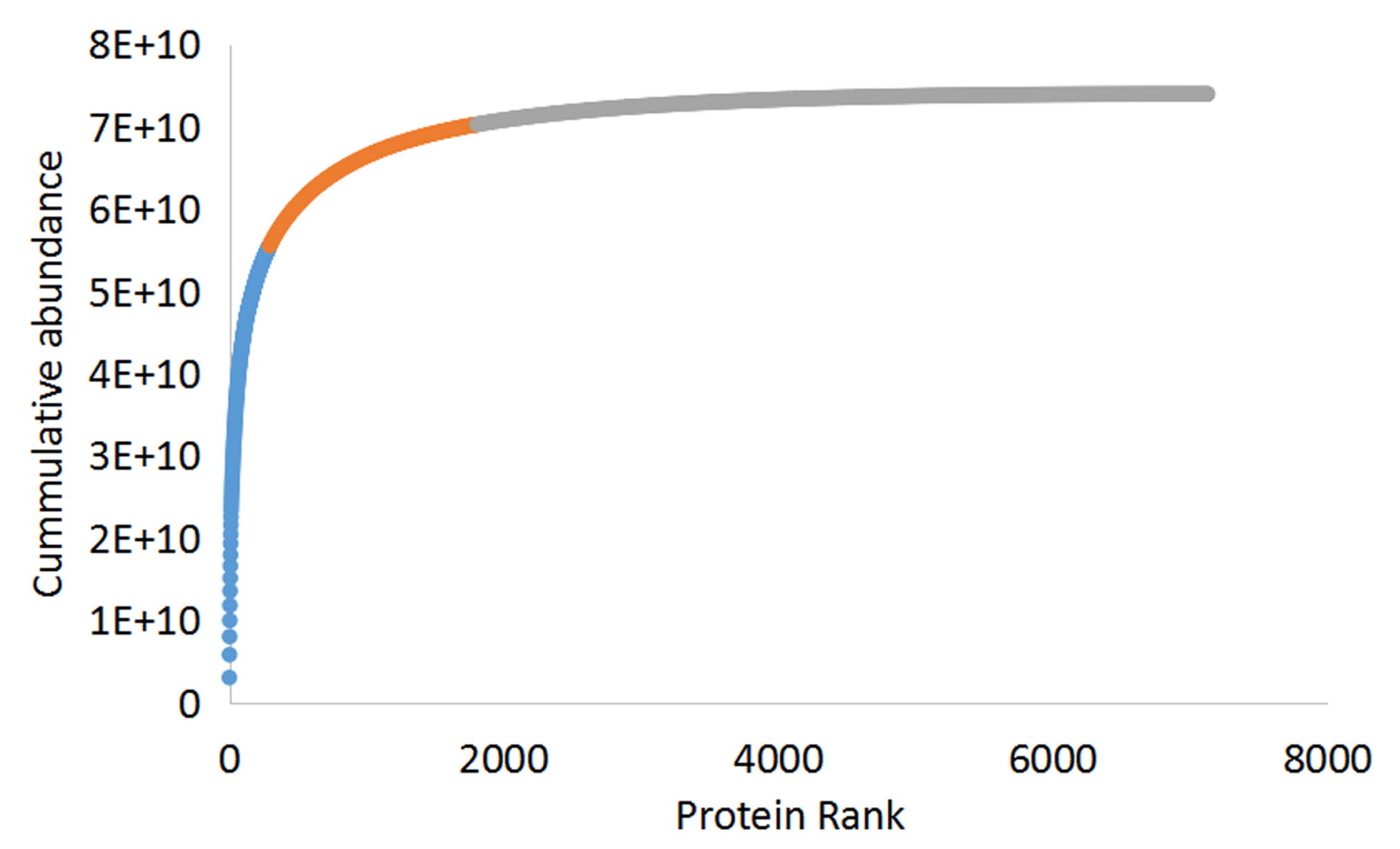

D

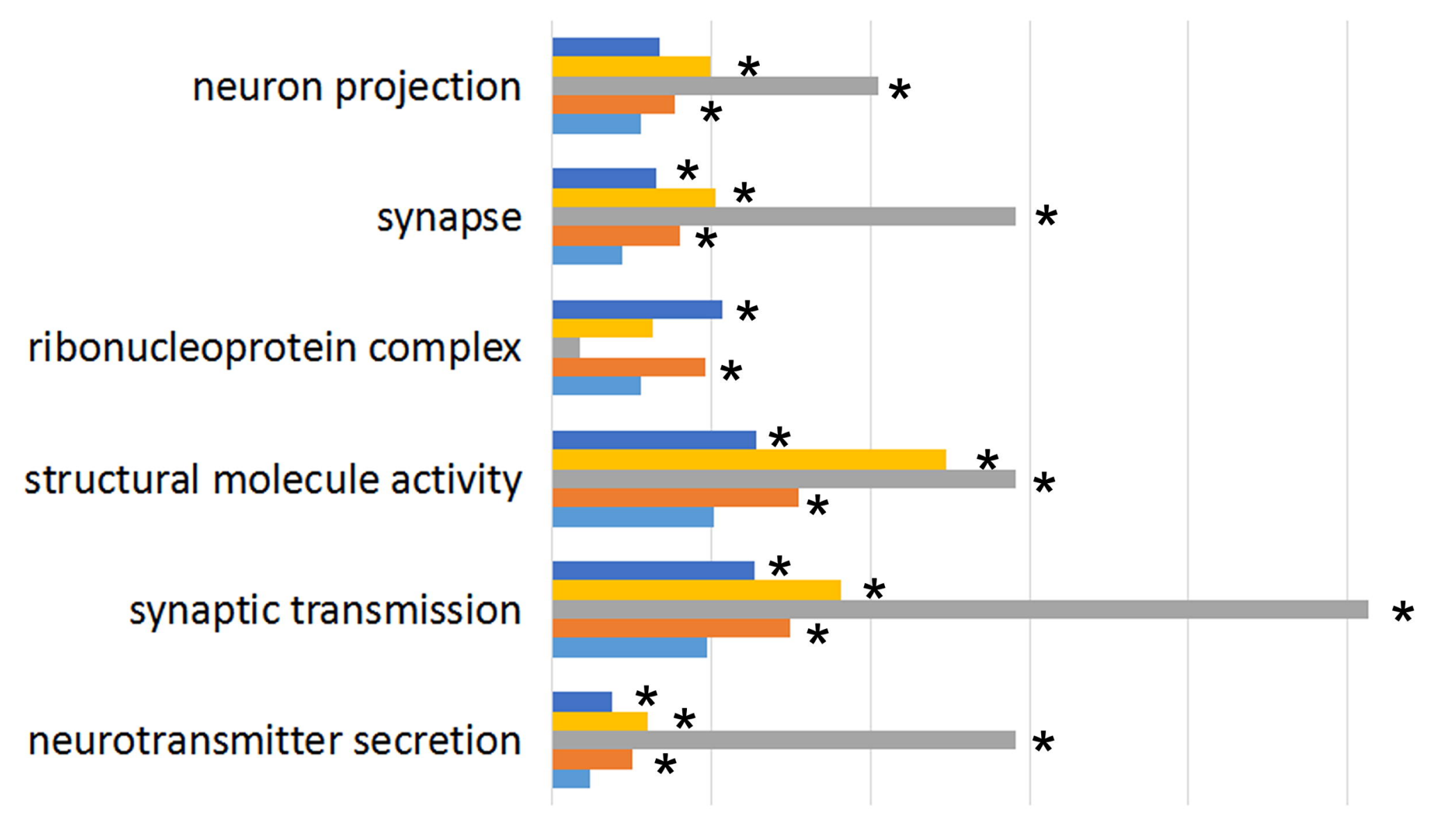

$\begin{array}{lllllll}0.00 \% & 2.00 \% & 4.00 \% & 6.00 \% & 8.00 \% & 10.00 \% & 12.00 \%\end{array}$ 
을

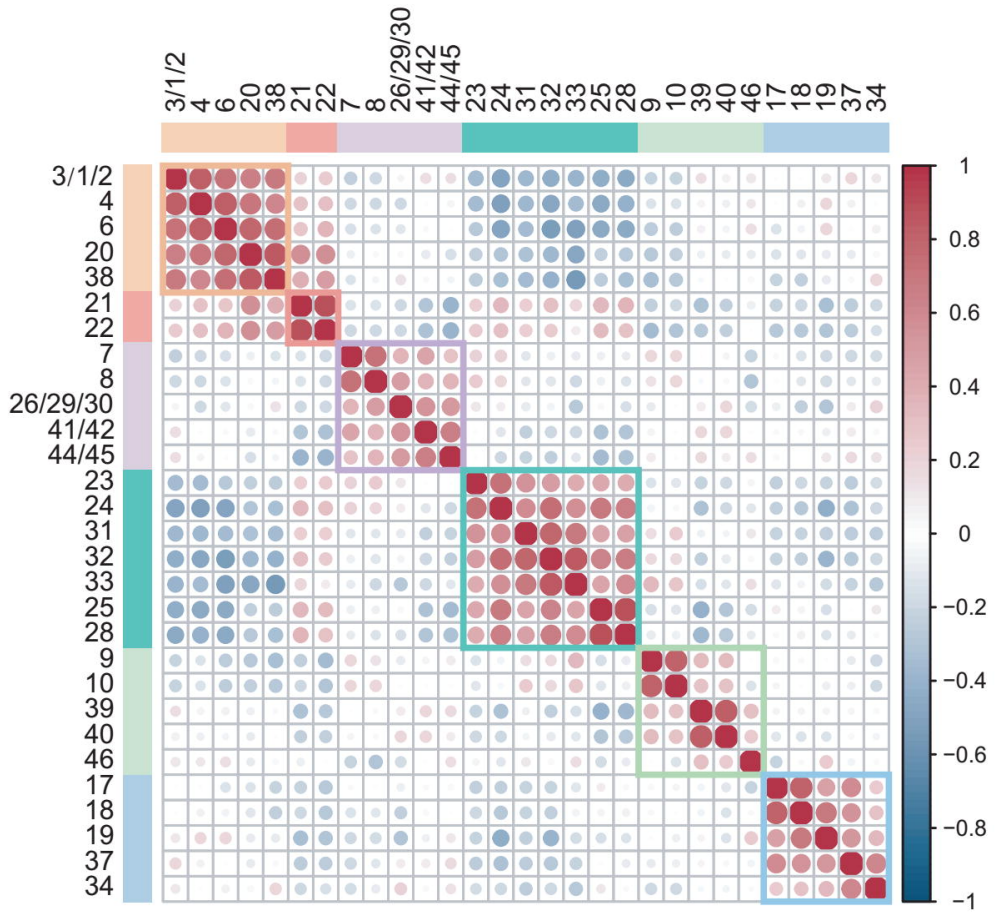

B

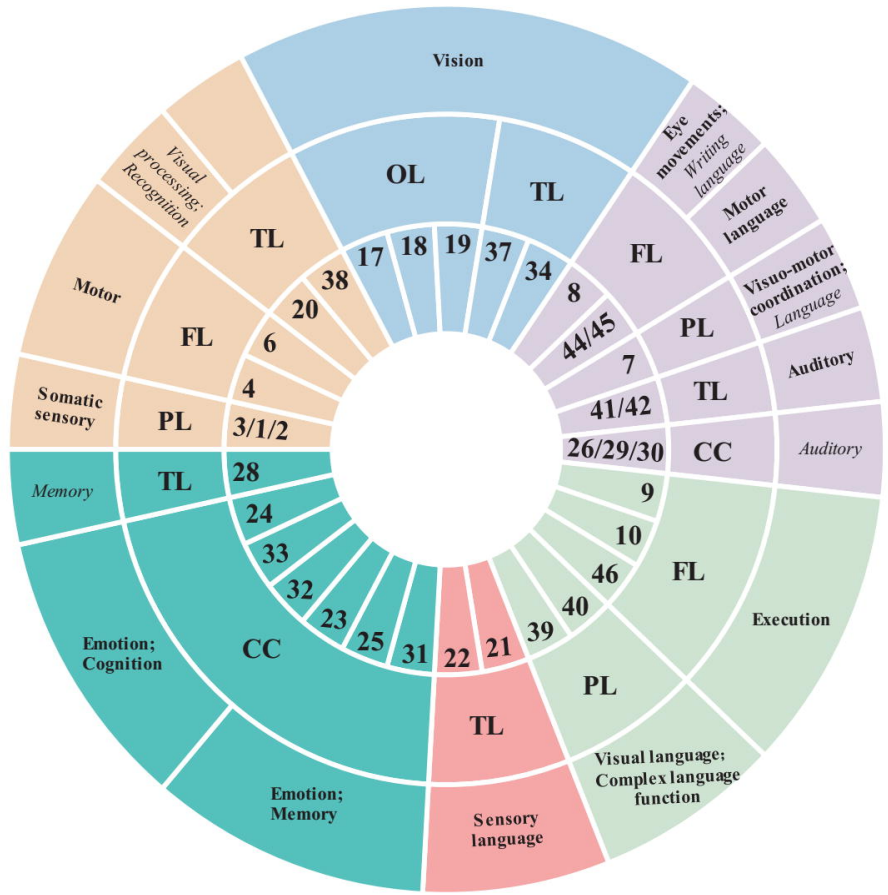

Lateral Surface

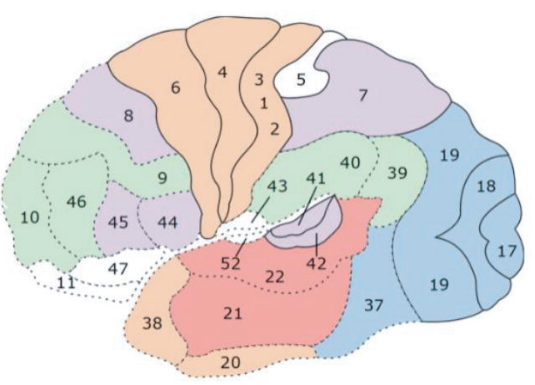

Medial Surface

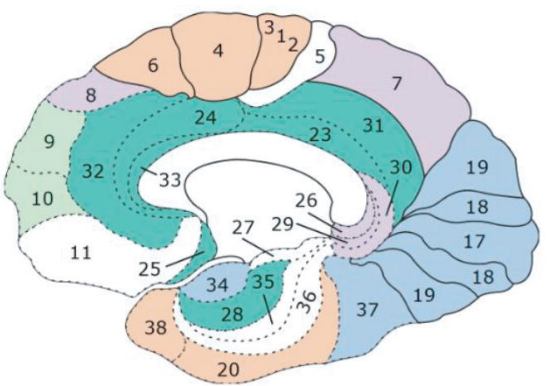

Cluster 1: motor and sensory cluster

- Cluster 2: Wernicke's area cluster

- Cluster 3: auditory and Broca's area cluster

- Cluster 4: cingulate cortex cluster

Cluster 5: hetrogenous function cluster

- Cluster 6: vision cluster

PL: Parietal Lobe

FL: Frontal Lobe

TL: Temporal Lobe

OL: Occipital Lobe

CC: Cingulate Cortex

Italic : noncanonical function

Bold : canonical function 
A

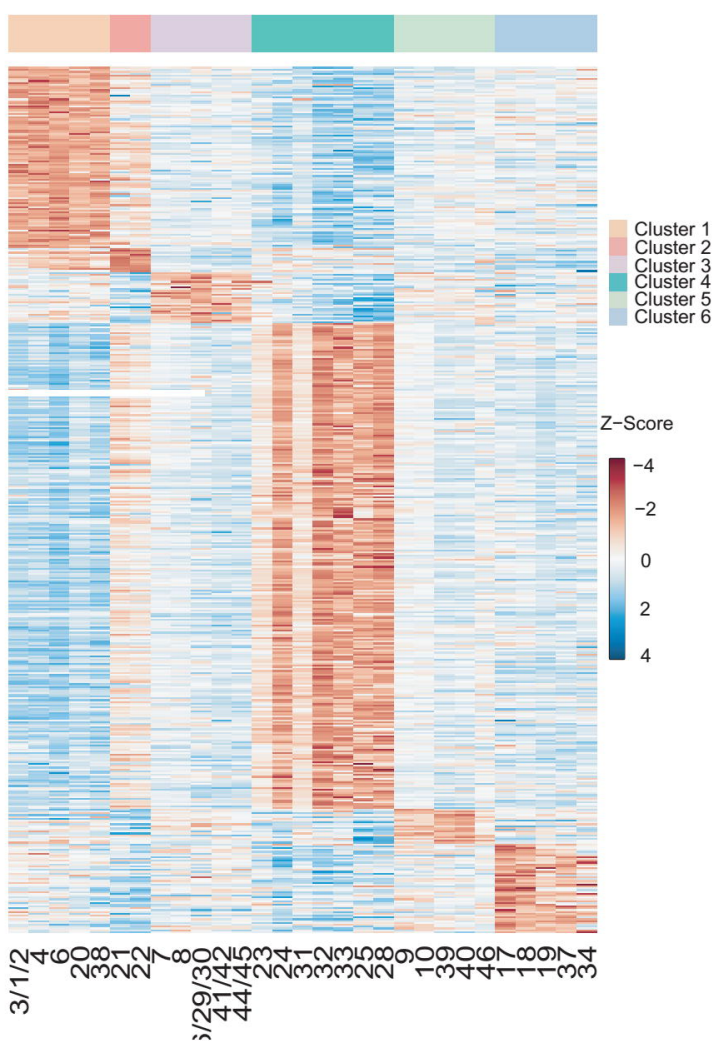

D

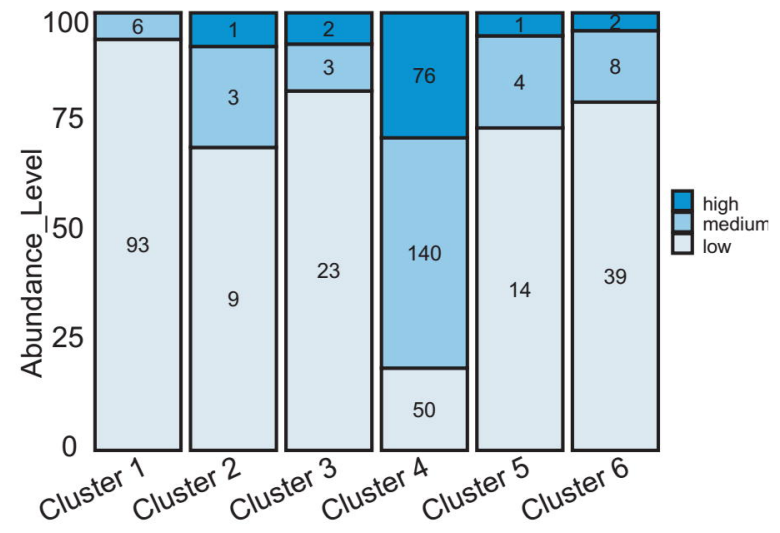

B
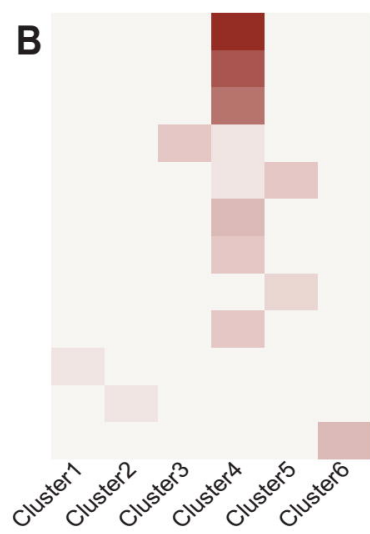

C

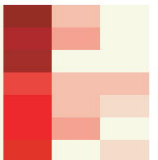

Huntington's Disease
Parkinson's disease

Progressive neurological disorder

Schizophrenia

Alzheimer disease

Dementia

Psoriasis

Epilepsy

Tauopathy

Mitochondrial disorder

Complex partial seizure

Migraines

Mania

Giant axonal neuropathy
Combined oxidative phosphorylation deficiency 32 Autosomal recessive LaFora epilepsy

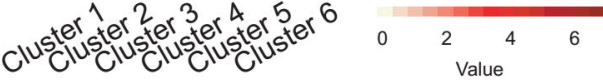

E

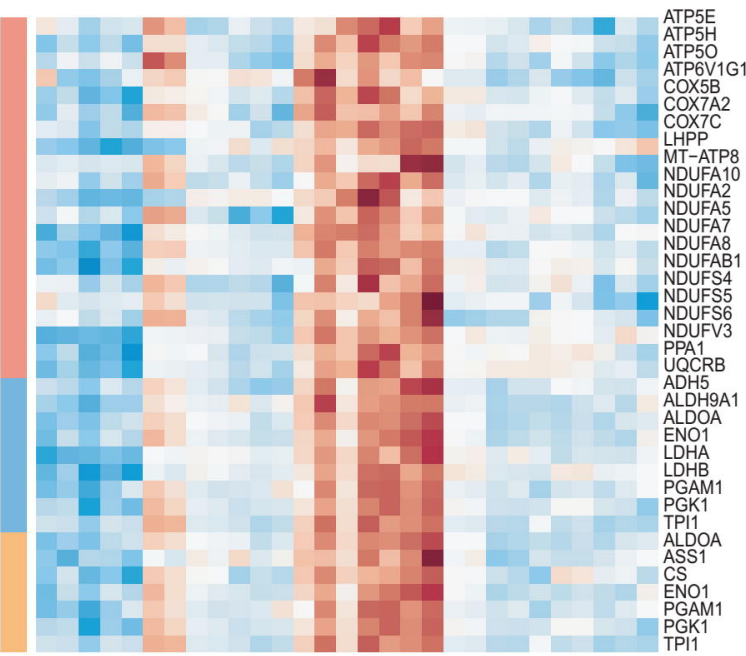

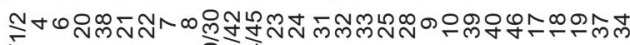

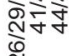

\&

Oxidative phosphorylation Glycolysis / Gluconeogenesis Biosynthesis of amino acids
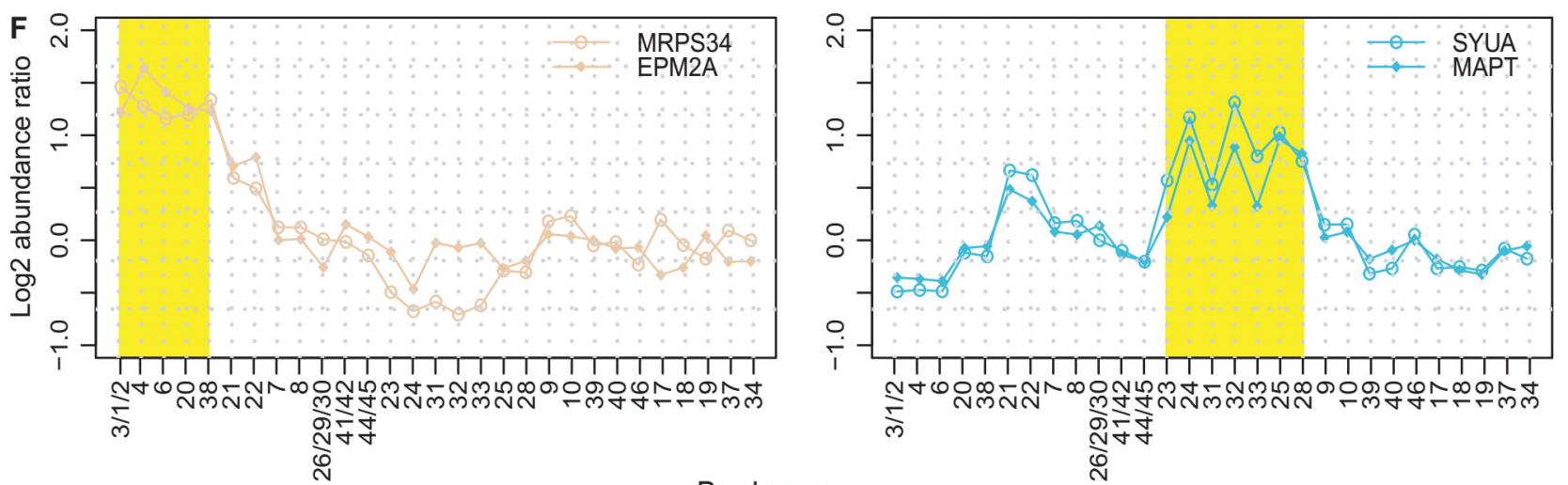

Brodmann area 


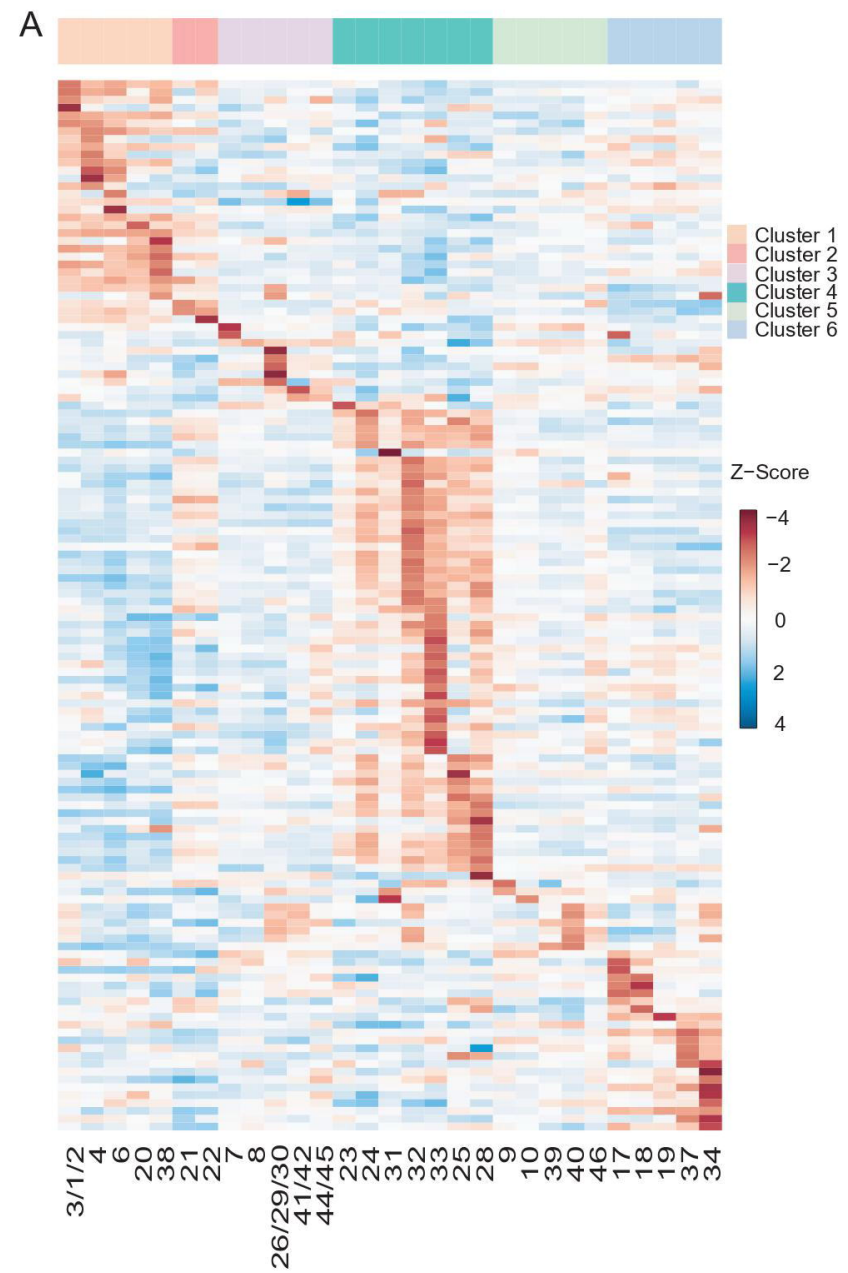

B
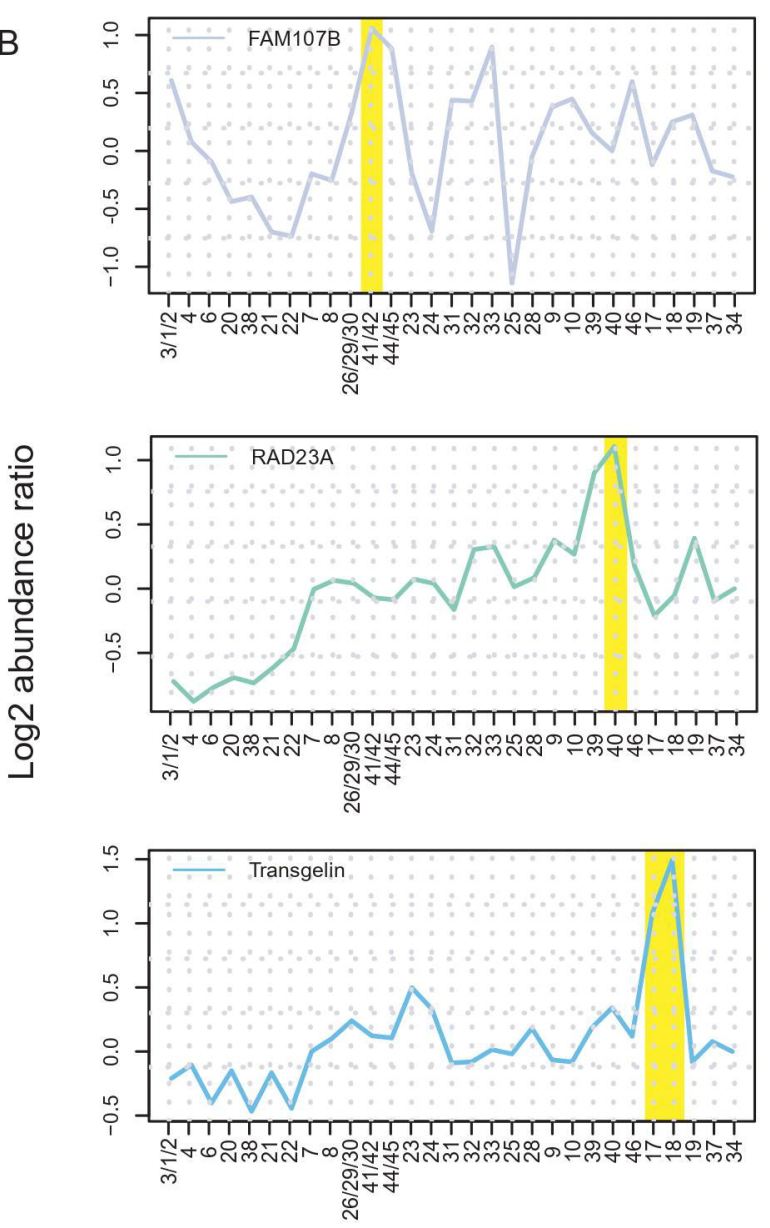

Brodmann area 
A

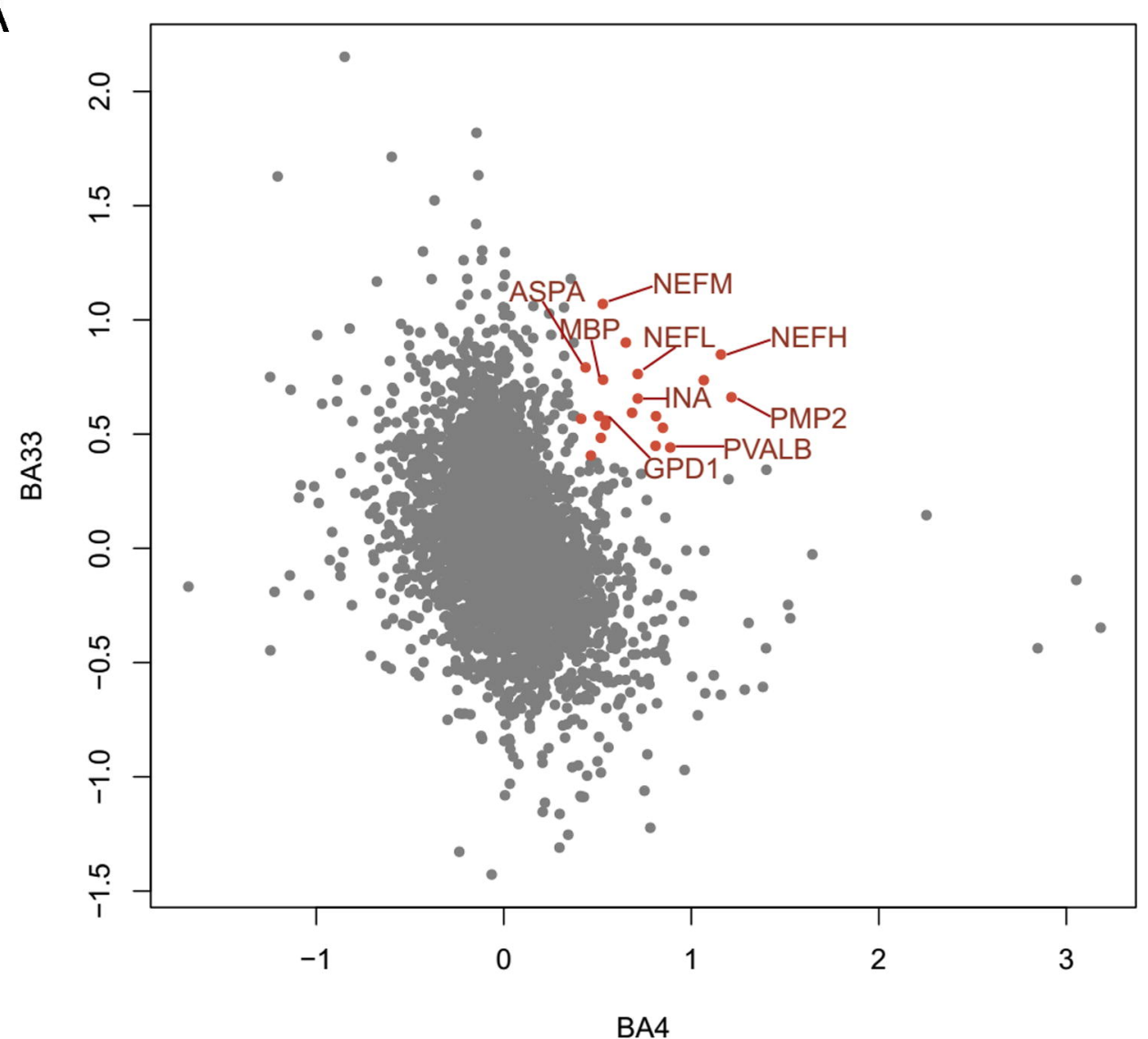

C

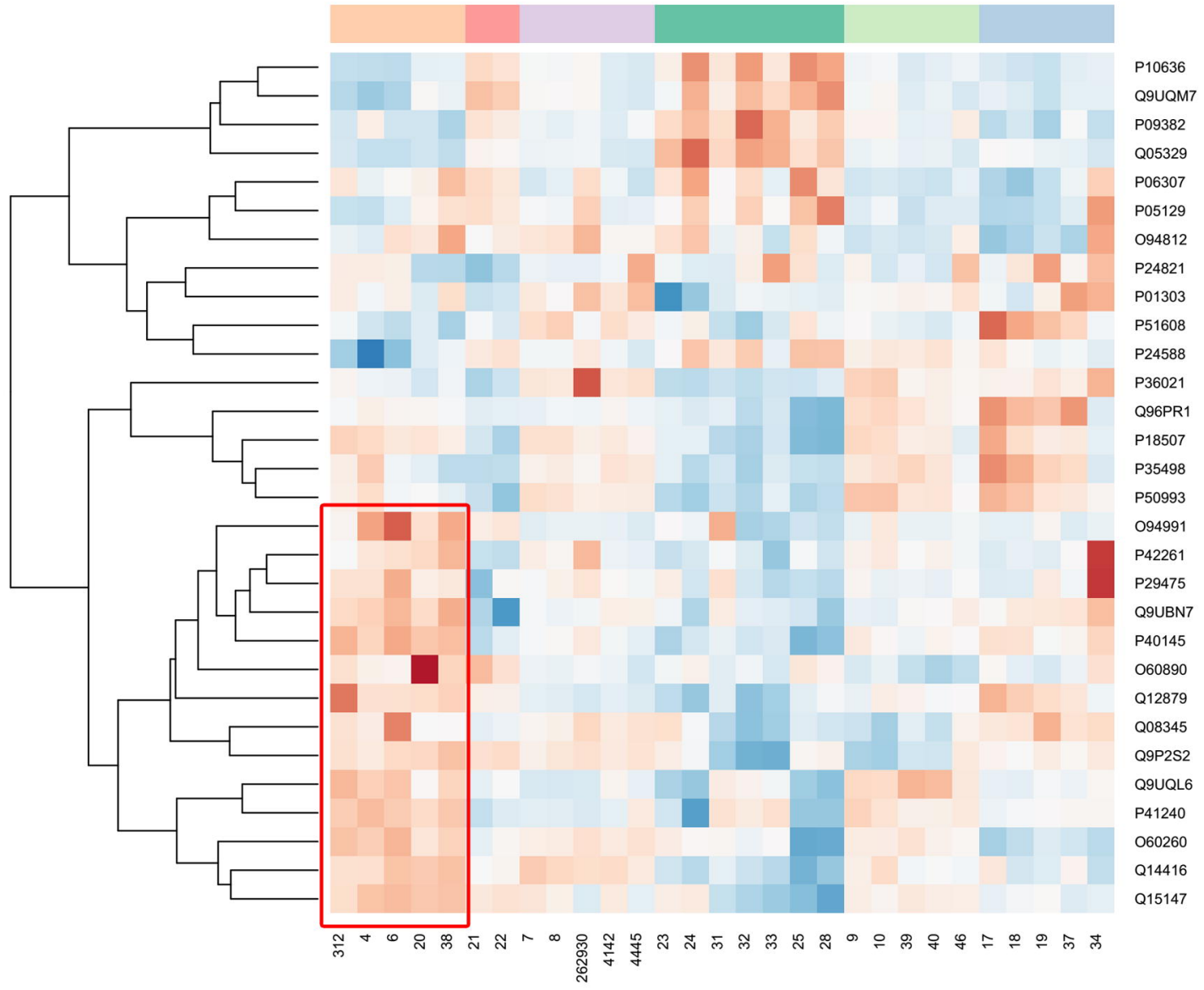

B

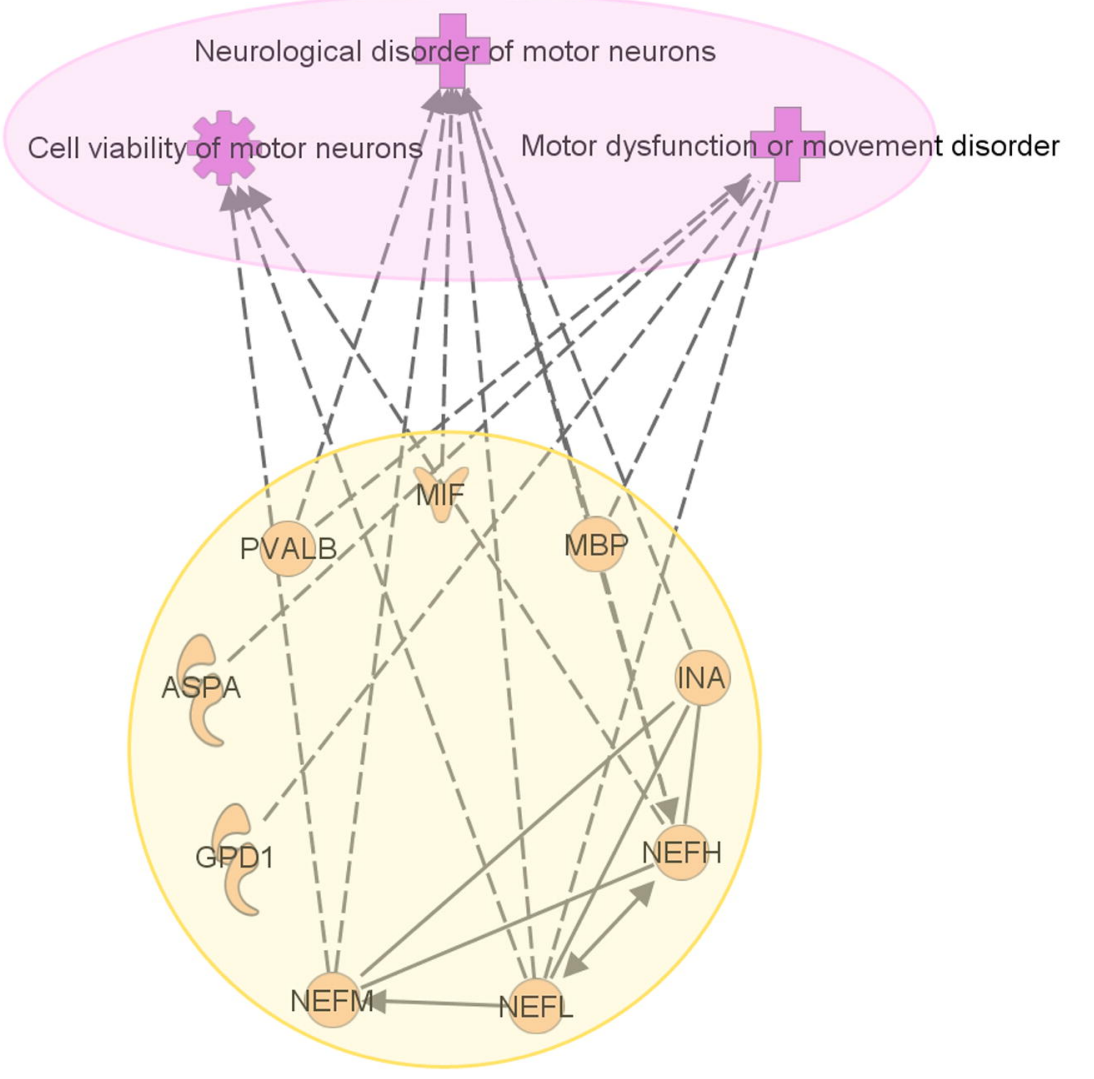

D

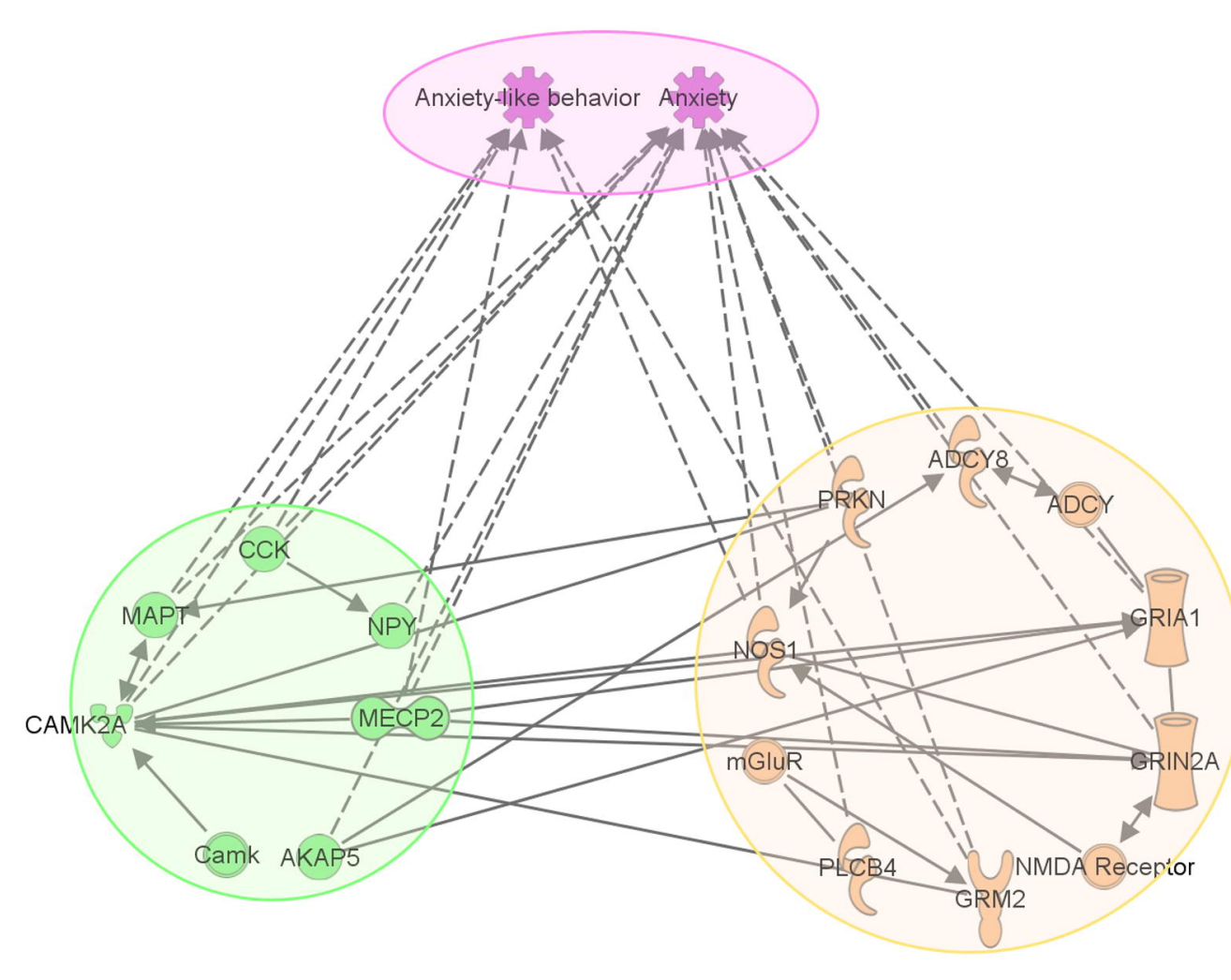


A

genes detected

as proteins

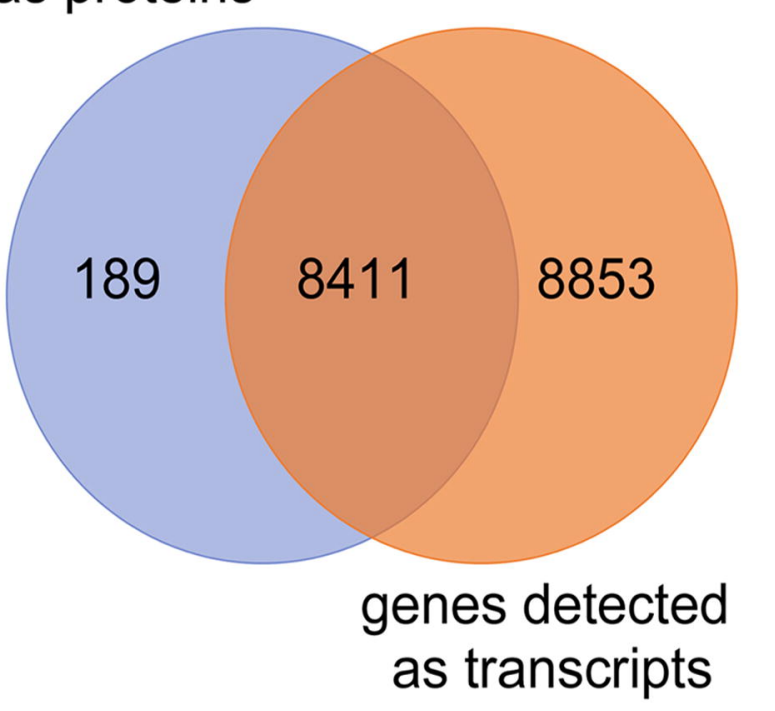

D

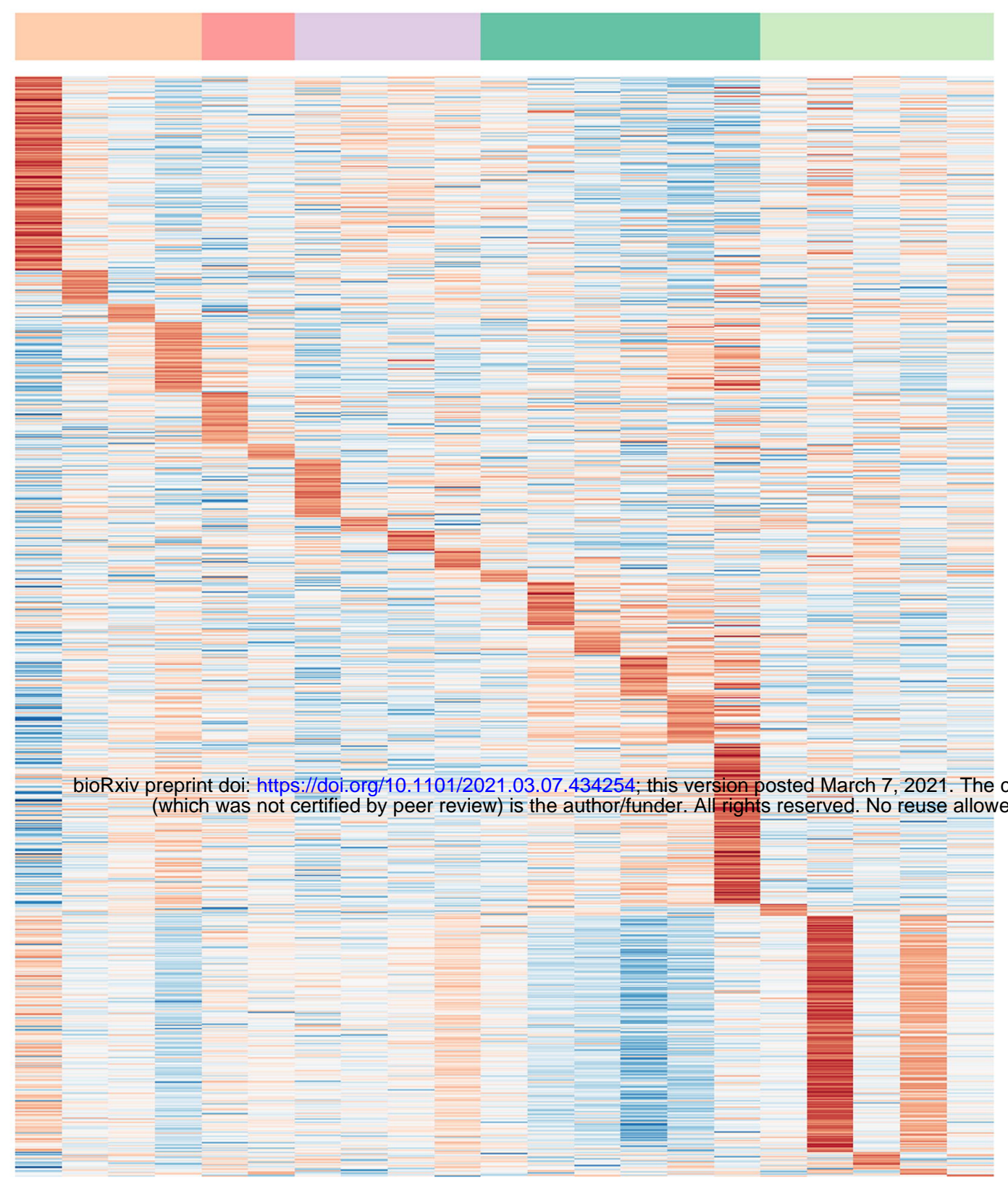

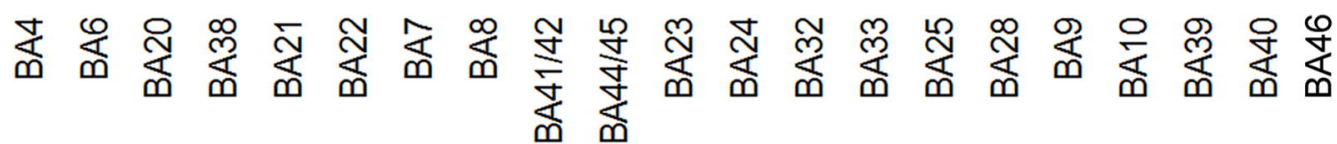

$\mathrm{F}$

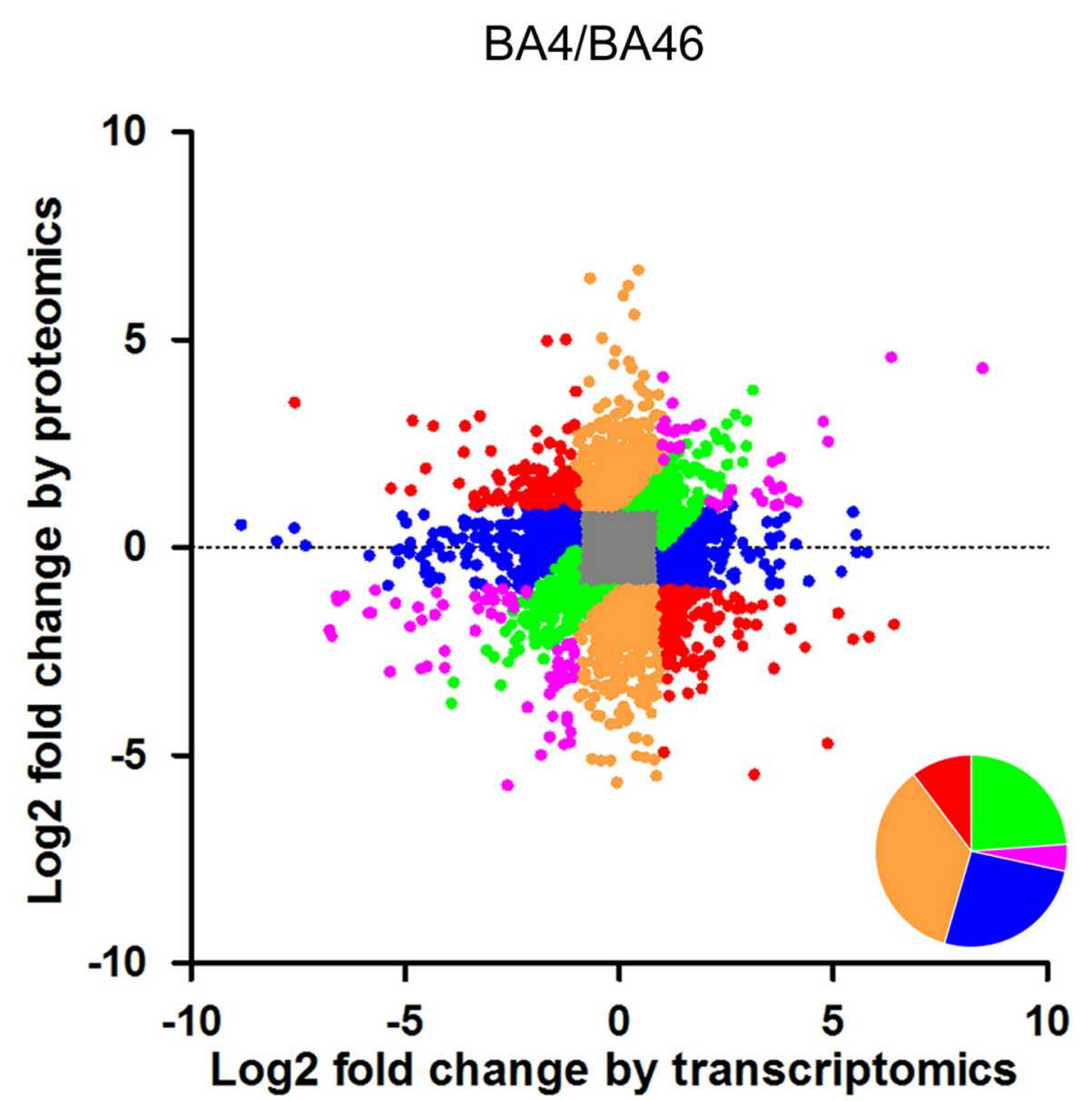

B

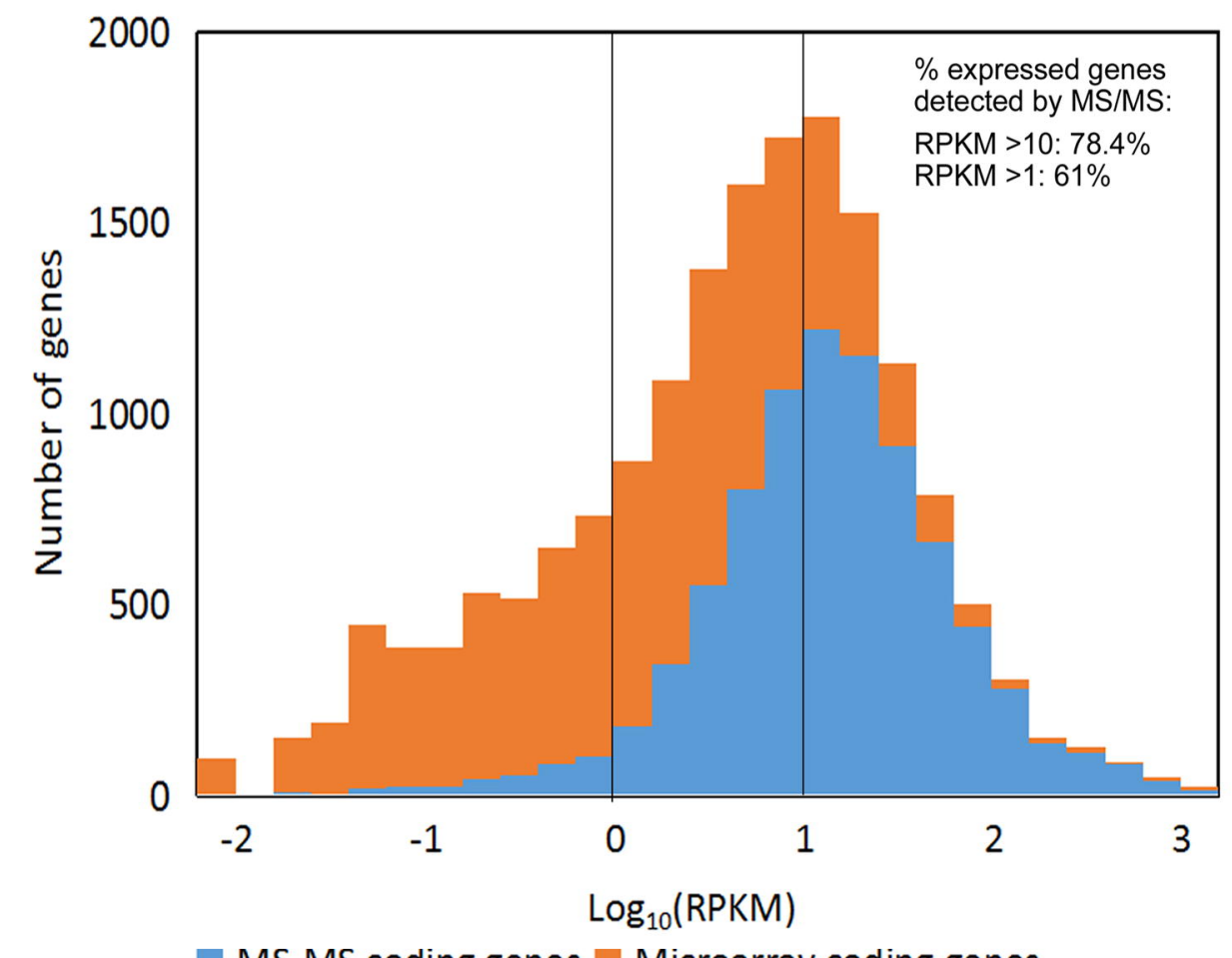

MS-MS coding genes $\square$ Microarray coding genes

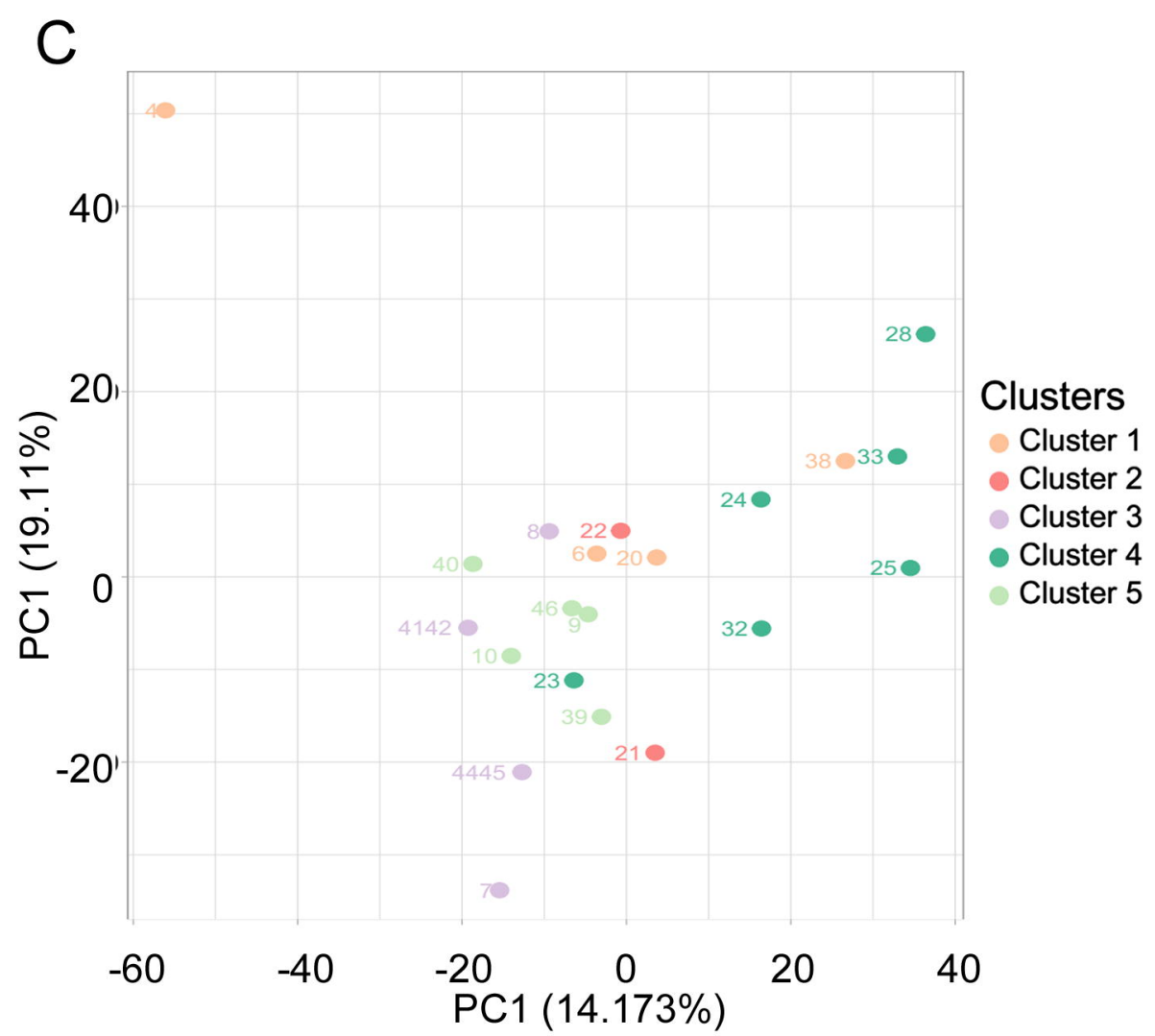

E

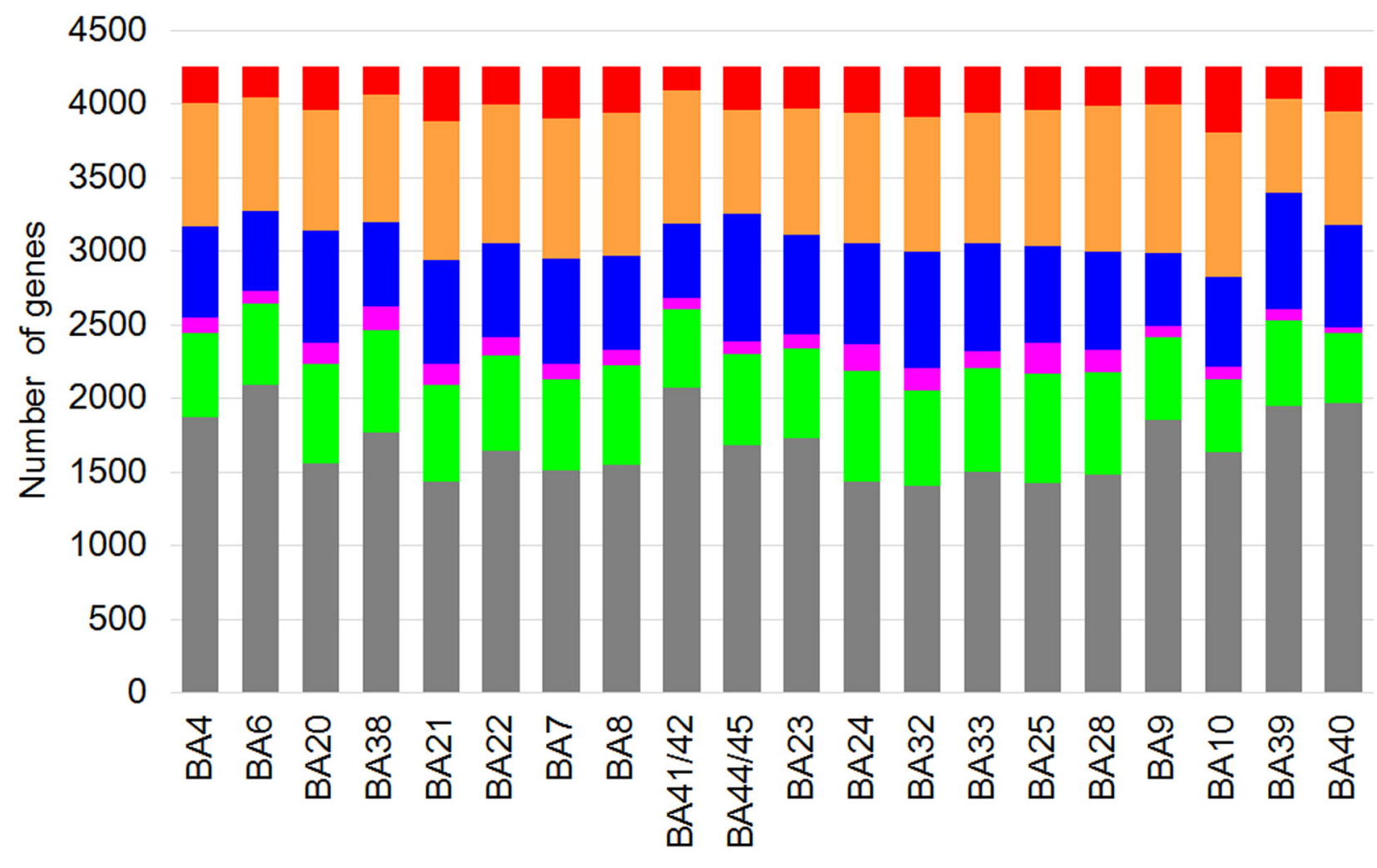

\section{G}
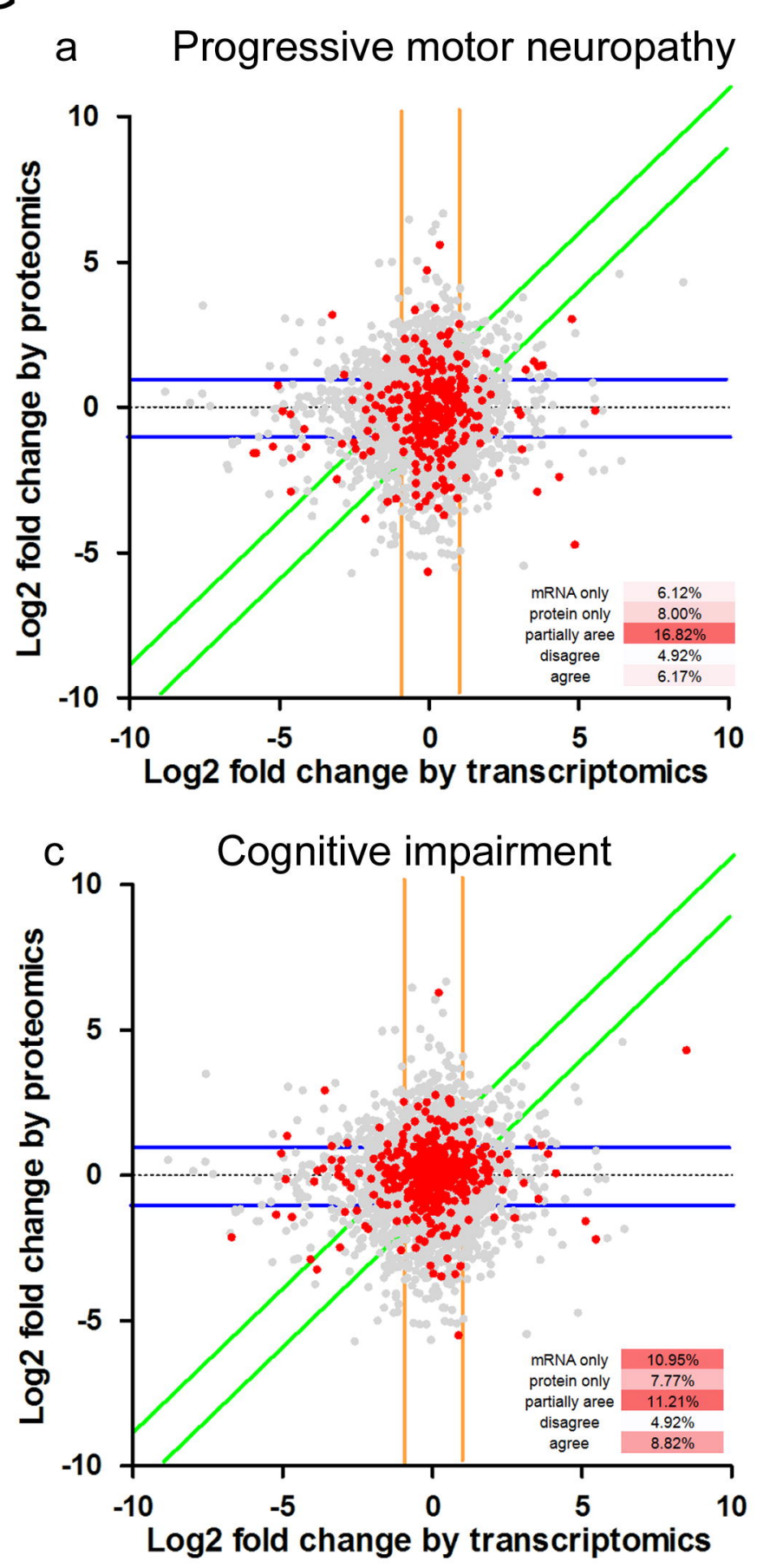
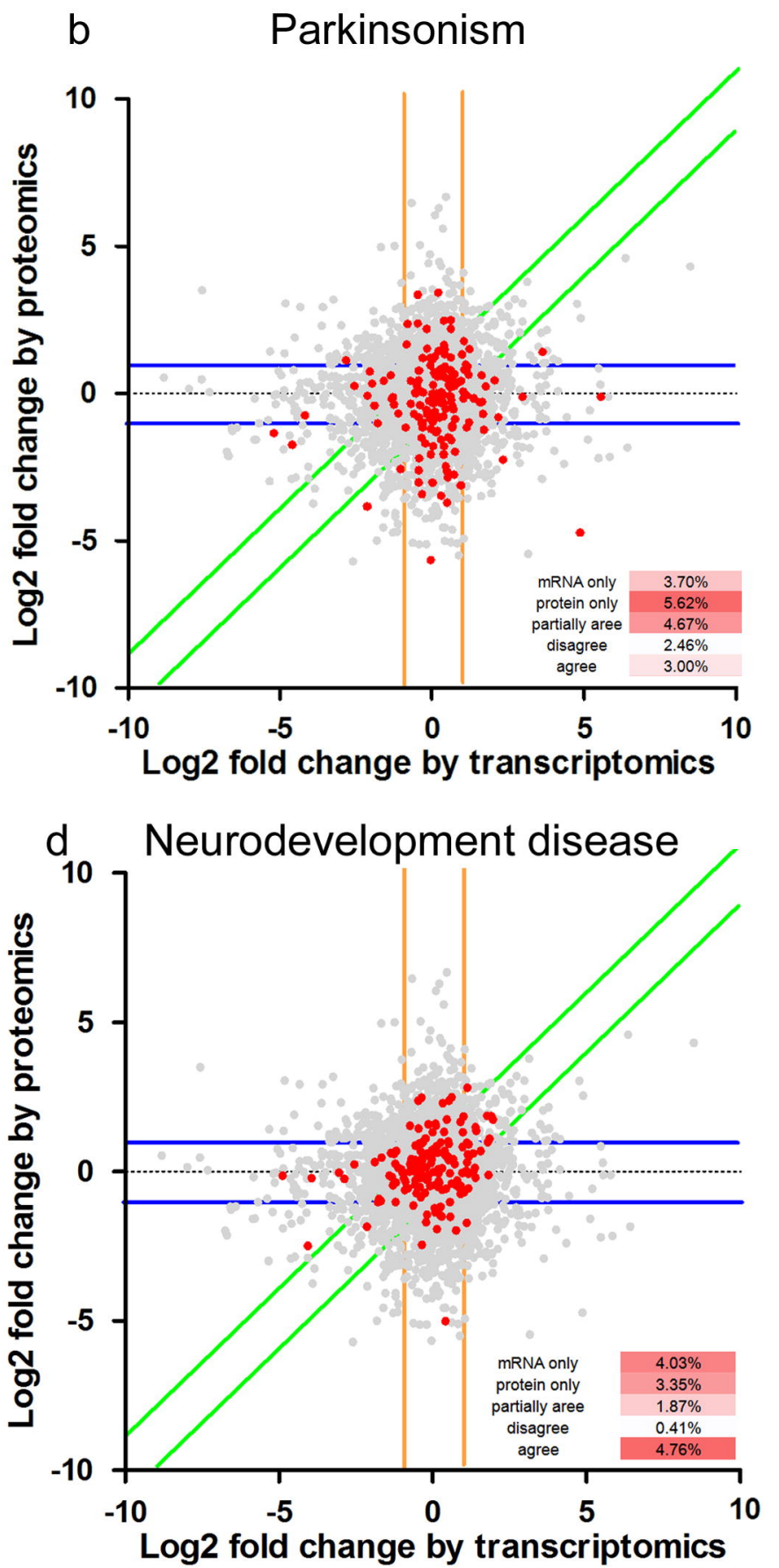